\title{
Criteria to distinguish between regional and contact zone monazite - a case study from Proterozoic North Delhi Fold Belt (NDFB), India
}

\author{
Department of Geology, University of Delhi, Delhi - 110007. *E-mail: pantnc@gmail.com
}

North Delhi Fold Belt (NDFB) is a part of a NNESSW trending Proterozoic mobile belt hosting sulfide mineralization in Khetri Copper Belt (KCB) towards its western margin. It preserves assemblages of amhibolite facies Barrovian metamorphism locally overprinted by a thermal metamorphic event. Monazite $\left(\mathrm{REEPO}_{4}\right)$ is developed in both the assemblages. Detailed chemistry and chemical geochronology of monazites in regional and contact metamorphic assemblages depict distinct chemical traits and ages. Two stage growth of metamorphic assemblages at 1100 Ma and $\sim 920 M a$ is recorded. Contact zone monazite grew at $\sim 650 \mathrm{Ma}$ under hydrothermal conditions. It has low thorium and is cerium enriched in comparison to the regional metamorphic monazite. Low Th and high Ce/Th ratio in contact zone monazite is proposed as discriminating criteria between regional and contact metamorphic monazites.

\section{Introduction}

Monazite is a natural rare earth element bearing monoclinic phosphate mineral having the general formula $\mathrm{REEPO}_{4}$. Substitutions are possible on both cationic and anionic sites. Cationic substitutions include trivalent substitutions (e.g. $\mathrm{La}^{3+}$ to $\mathrm{Gd}^{3+}$ ), $\mathrm{A}^{+}$to $\mathrm{REE}^{3+}$ (e.g. $\mathrm{Na}, \mathrm{K}$ to $\mathrm{REE}^{3+}$ ), substitution by tetravalent (U4+, Th4+) cations for trivalent REE $\left(4 \mathrm{REE}^{3+}=3 \mathrm{~B}^{4+}+\right.$ ?), coupled substitutions of trivalent REE with divalent and tetravalent cations $\left(2 \mathrm{REE}^{3+}=\mathrm{A}^{2+}+\mathrm{B}^{4+}\right.$ wherein $\mathrm{A}$ can be $\mathrm{Ca}$ and $\mathrm{B}$ is often Th in natural monazites) while substitutions on anionic sites can result in transformation to vanadates, arsenates or chromates (Kolitsch and Holstam, 2004). Besides, coupled substitutions on the cationic as well as the anionic groups occur which have been experimentally investigated in the huttonite/ thorite-Ce monazite-xenotime $\left(\mathrm{ThSiO}_{4}-\mathrm{CePO}_{4}-\mathrm{YPO}_{4}\right)$ system and a miscibility gap between monazite-xenotime and temperature dependence of substitution demonstrated (Seydoux-Guillaueme, et al. 2002). Monazite is a common accessory mineral in metamorphic rocks, it strongly influences REE and Th-U budget of these rocks and is highly useful in dating of metamorphic events on account of very low inherited $\mathrm{Pb}$ (Copelend et al., 1988). Low rate of $\mathrm{Pb}$ diffusion in monazite lattice has been considered suitable of its utility in deciphering the peak metamorphic conditions (Janots et al, 2008, 2009). Conditions of metamorphism of multiple phases can be ascertained using mineralogical and textural criteria. Monazite composition readjusts to the changing P-T conditions and these variations preserve the evolutionary history of a terrain (e.g. Zhu and O’Nions, 1999; Krenn and Finger, 2007). Studies on control of monazite composition in metamorphic rocks are relatively scarce though monazites developed in contact metamorphic aureole were described as depleted in europium compared to those produced as a product of regional metamorphism (Rosenblum and Mosier, 1983) in a study on monazite grains recovered from the heavy mineral fractions.

Khetri copper belt is the northern most entity of North Delhi Fold Belt (NDFB) comprising two metamorphic series representing two episodes of prograde regional metamorphism i.e. andalusite and sillimanite in the north and kyanite sillimanite in the south separated by an east west trending fault between Dhanota and Chappoli (Dasgupta, 1964, 1968; Lal and Shukla 1975; Sharma 1988). Several models of genesis of ore in NDFB ranging from syn-sedimentary deposition and metamorphism (Sarkar and Dasgupta, 1980; Basu, 1986), hydrothermal (Roy Choudhury and Das Gupta, 1965, Banerjee, 1976) and post-metamorphic Iron oxide-Copper-Gold (IOCG) type (Knight et al., 2002) have been proposed.

In the present work we describe texturally constrained monazite from regional metamorphic as well contact metamorphic association from the rocks of NDFB and discuss control on monazite chemistry in these two situations.

\section{Geological setting}

Delhi fold belt has been divided into two parts NDFB and SDFB (Sinha-Roy et al; 1998). North Delhi Fold Belt (NDFB) which is characterized by several fossil grabens, horsts, distributed broadly in three main sedimentation sub- basins (Singh, 1984). From east to west, these are the Bayana- Lalsot, the Alwar, and the Khetri subbasins. Polyphase deformation in the Alwar basin has resulted in complex outcrop patterns with abundant structural duplication. The rocks are folded into appressed upright to isoclinals shallow to moderate plunging NNE-SSW trending antiforms and complementary synforms, which were subsequently refolded on NW-SE and ENE- WSW axial planes, resulting in axial culminations and depressions. Based on the occurrence of andalusite- staurolite-biotite-muscovite association, Sharma (1988) estimated $500-550^{\circ} \mathrm{C}$ temperature and 3-4 kbar pressure for Alwar basin metamorphism. Monazite bearing samples in the present study are reported from two locations. Khetri copper 
mine within the Khetri Copper Belt (KCB) is located $\sim 25 \mathrm{~km}$ northwest of Golwa-Gangutana area.

Khetri Copper Belt: The Khetri Copper Belt constitutes the northernmost entity of the Aravalli craton and extends for about 80 $\mathrm{km}$ from Singhana (Jhunjhun District) in the NE to Sangarva (Sikar District) in the SW. The belt is further separated into northern and southern parts by the NW-SE striking transverse Kantli Fault (Gupta et al. 1998). Following Heron (1923), Das Gupta (1968) considered that the Khetri Copper Belt is made up of rocks of the Delhi Supergroup, which are further divided into (1) an older psammitic dominated Alwar Group and (2) a younger pelitic dominated Ajabgarh Group. In contrast, some later studies consider rocks of the Khetri Copper Belt as pre - Delhi (Chakrabarti \& Gupta, 1992). The NNESSW to NE - SW striking metasedimentary rocks of the northern Khetri Copper Belt consists of feldspathic quartzite with magnetite, banded amphibole quartzite, garnetiferous chlorite schists, mica schists and quartzite, which are folded into a number of regional anticlines and synclines (Das Gupta, 1968). In contrast, Sarkar and Dasgupta (1980) and Sarkar (2000) concluded that these rocks belong to a homoclinal sequence and are part of one limb of a regional fold. The Khetri Copper Belt displays two episodes of prograde regional metamorphism $\left(\mathrm{M}_{\mathrm{I}}\right.$ and $\left.\mathrm{M}_{2}\right)$, the andalusite - sillimanite facies in the north and a transition to kyanite - sillimanite facies towards the south, and there is an eastward increase in the grade of metamorphism (Lal

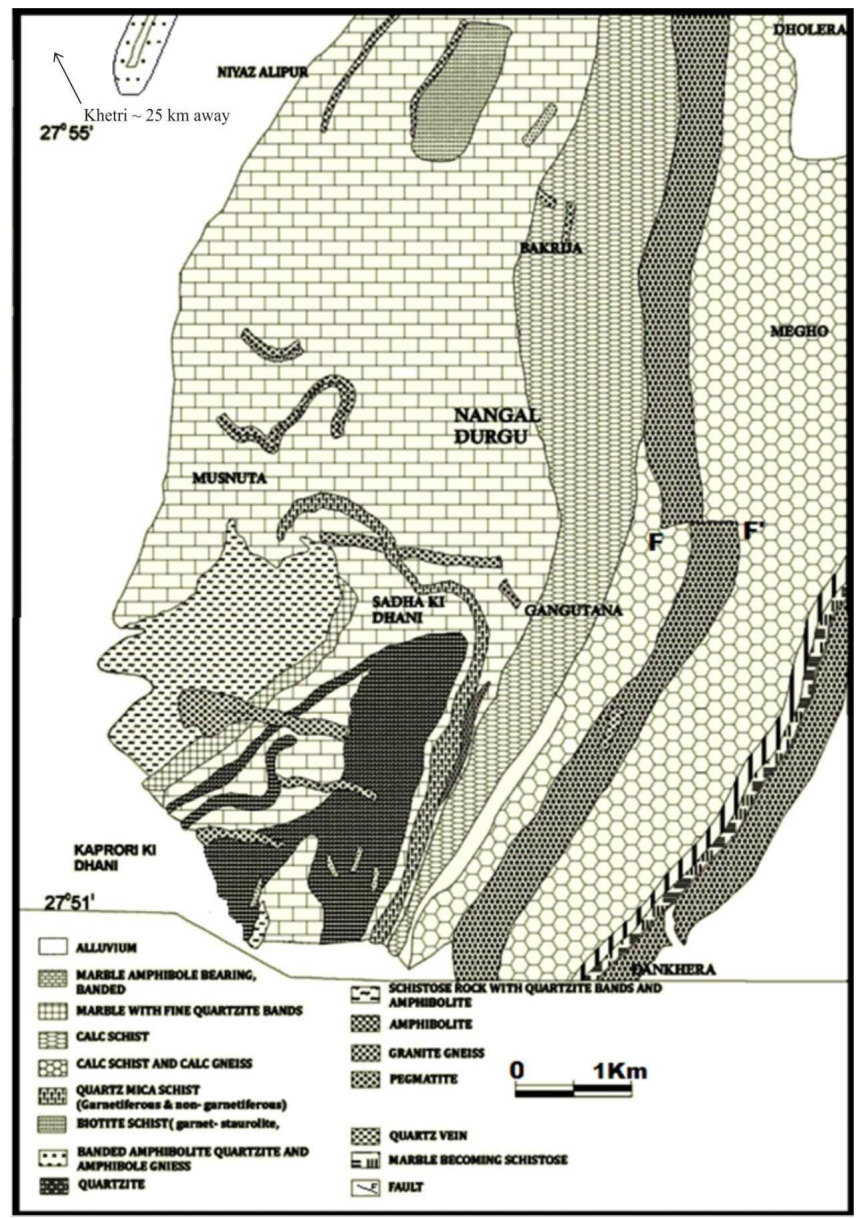

Fig.1. A geological map of the area around Narnaul, Mahendergarh district, Haryana. (Bhola, 1989). Here, metapelites occur as a $\sim 500 \mathrm{~m}$ thick band of $\sim 6 \mathrm{~km}$ strike length near Golwa village. and Shukla, 1975; Lal and Ackermand, 1981). The intrusive rocks in the KCB are largely represented by mafic and granitoids rocks. Ray identified a $170 \mathrm{~km}$ long NNE- SSW trending lineament, the albitite line in the region. Besides, minor amount of felsic volcanic have also been reported.

Golwa Gangutana area: The rocks of Alwar and Ajabgarh Groups of Delhi Supergroup are exposed in southern part of Haryana state and adjacent part of Rajasthan. Quartzite and metamorphosed mafic volcanics represent the Pratapgarh Formation of Alwar Group while interbedded calc silicates, metapelites and arenites constitute the Kushalgarh Formation of Ajabgarh Group (Prasad et al., 1994). Metapelites occur as a $\sim 500 \mathrm{~m}$ thick band of $\sim 6 \mathrm{~km}$ strike length near Golwa village (Fig. 1) within the core of the refolded synform (Kundu et al., 2004).The emplacement of most of the granitoid plutons was structurally controlled, and they occur in the cores of anticline or parallel to foliation of the country rocks. These plutons have been interpreted as syn- to post- kinematic with respect to different episodes of the Delhi orogeny. Granitic activity in the KCB is considered to have taken place during the culmination of regional metamorphism.

\section{Petrography}

Several thin sections of mineralized rock and metapelites from Khetri and Golwa Gangutana were studied using polarizing microscope and scanning electron microscopy (SEM) to select suitable
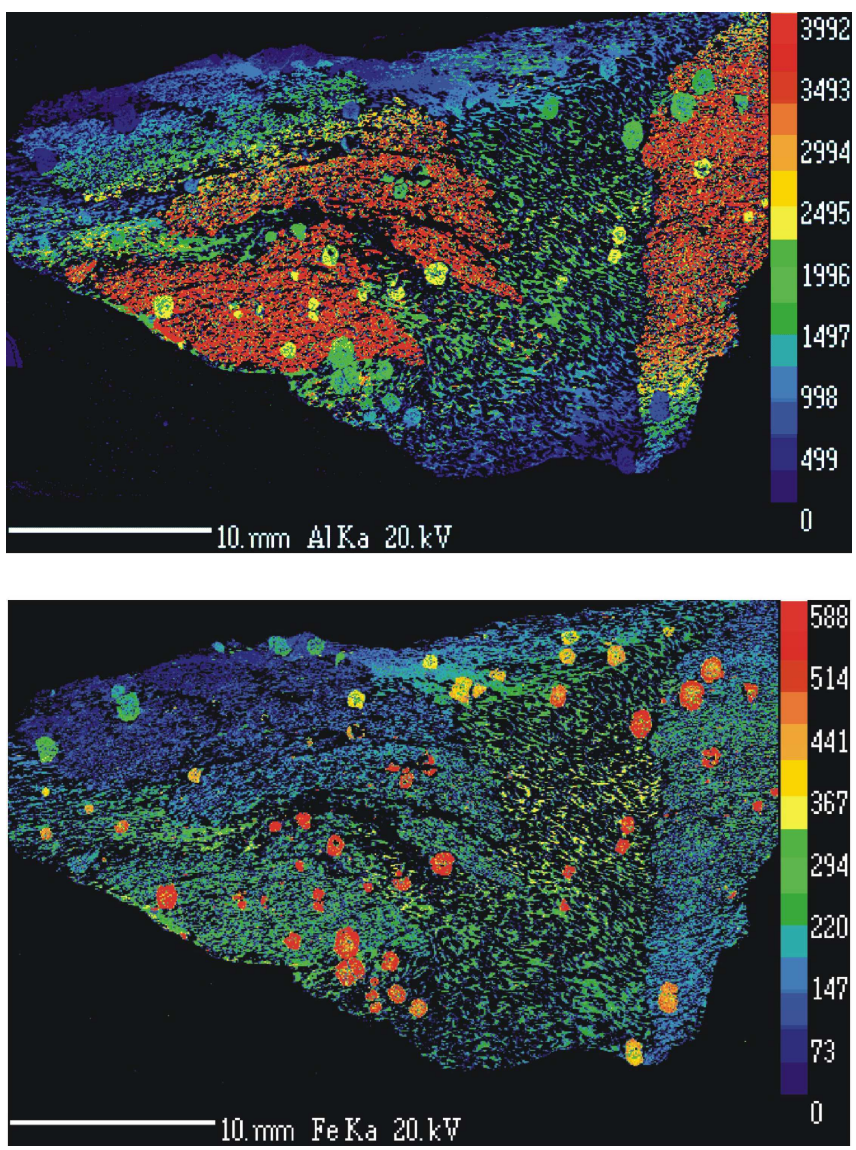

Fig.2. Thin section scale elemental X-ray maps of Al and Fe show presence of zoned as well as unzoned garnets. Porphyroblasts of staurolite and garnet as dominant constituent along with biotite and quartz and sub-ordinate muscovite and plagioclase. 
samples for investigating the monazite. Thereafter, one sample from garnet-staurolite schist and one sample from chlorite schist were chosen for detailed analysis. Sample GG-5 was collected from metapelites (garnet-staurolite schist) of Kushalgarh Formation and sample KH-2 was from chlorite schist of Khetri Copper Belt.

GG-5 is strongly foliated garnet-staurolite schist comprising porphyroblasts of staurolite and garnet as dominant constituent along with biotite and quartz and sub-ordinate muscovite and plagioclase (Fig. 2). Ilmenite, apatite and monazite are present as accessory phases. Schistosity is defined by aligned biotite and muscovite flakes and quartz grains. Staurolite occurs as the coarsest sub-idioblastic to idioblastic poikiloblasts and has inclusions of variable size. Garnet, quartz, micas and ilmenite are present as major inclusions in staurolite. The internal schistosity of staurolite is in continuation with external schistosity indicating it to be post-kinematic. Thin section scale elemental X-ray maps of $\mathrm{Al}$ and Fe show presence of zoned as well as unzoned garnets (Fig. 2). Garnet grains are present as inclusions in staurolite as well as in the matrix. Overgrowth as well as diffusion related zoning is inferred in garnets wherein the former appear to be an expression of garnet in two different P-T conditions thereby indicating polyphase metamorphism (Fig. 3). Peak metamorphic conditions (temperature around $645^{\circ} \mathrm{C}$ and pressure $7 \mathrm{kbar}$; Kundu et al., 2004) have been reported from this area.

$\mathrm{KH}-2$ is chlorite schist where biotite and chlorite define the foliation. It contains chlorite, biotite, cordierite, muscovite, quartz, garnet and $\mathrm{Cu}$ and $\mathrm{Fe}$ - sulfides as major metamorphic assemblage.
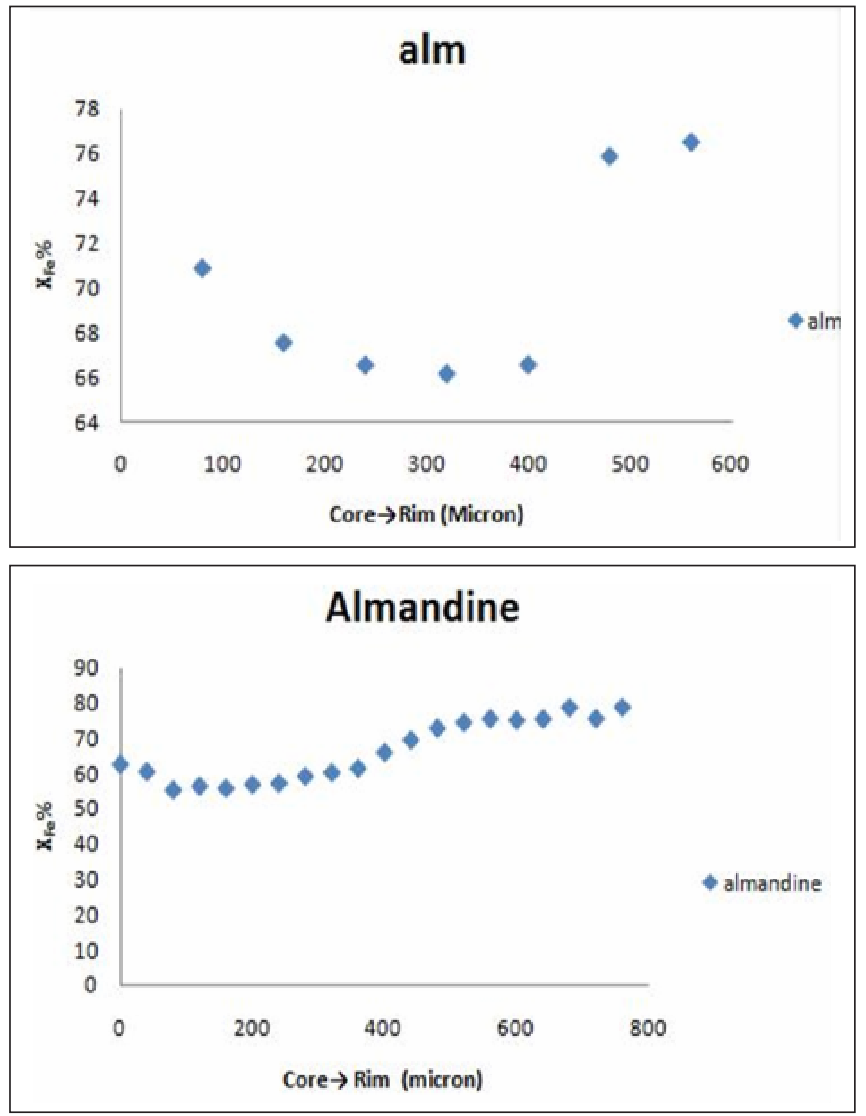

Fig.3. Overgrowth as well as diffusion related zoning is inferred in garnets wherein the former appear to be an expression of garnet in two different P-T conditions thereby indicating polyphase metamorphism.
Chalcopyrite and pyrrhotite are present as main sulfide phases and occur typically as hydrothermal vein fillings (Fig. 4). Monazite is associated with these hydrothermal veins (Fig. 4). The rock has retrogressed chlorite formed from biotite and garnet. Biotite is of two generations with the earlier being commonly retrogressed to chlorite and the latter occurring at the margins of chlorite in association with cordierite (Fig. 5). This association and texture is inferred as evidence of thermal metamorphism with following operative reaction.

$$
\text { Chlorite }+ \text { muscovite }=\text { cordierite }+ \text { biotite }+\mathrm{H}_{2} \mathrm{O}
$$

Temperature of $\sim 600^{\circ} \mathrm{C}$ is inferred for development of cordierite+ biotite in a mineralized contact aureole (Whylidal et.al, 2009)

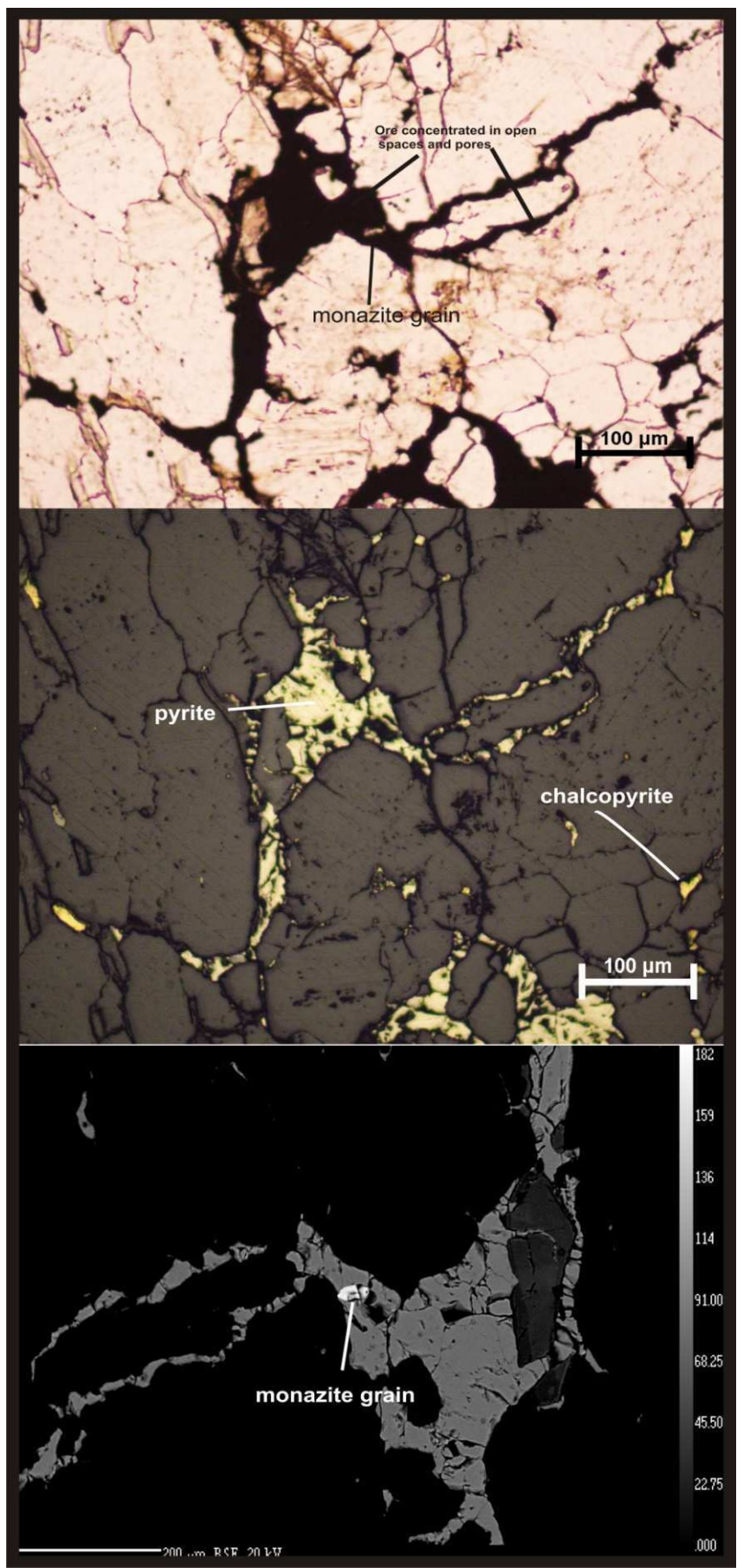

Fig.4. Chalcopyrite and pyrrhotite are present as main sulfide phases and occur typically as hydrothermal vein fillings. 


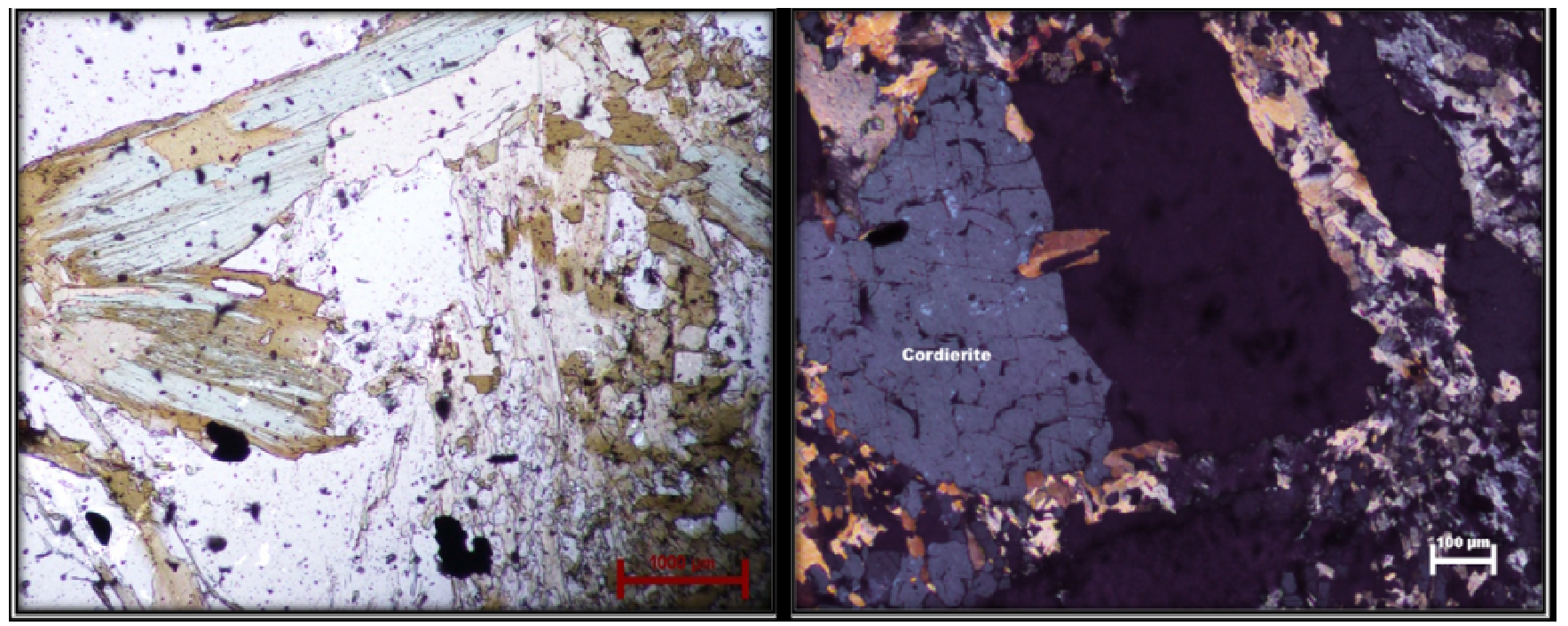

Fig.5. Biotite is of two generations with the earlier being commonly retrogressed to chlorite and the latter occurring at the margins of chlorite in association with cordierite.

\section{Analytical conditions}

The monazite analysis work was carried out on a CAMECA SX100 Electron Microprobe at EPMA Laboratory, Geological Survey of India, Faridabad and IIT Khadagpur. The analyses were carried out at $20 \mathrm{kV}$ and $200 \mathrm{nA}$ beam current. $\mathrm{Pb}(\mathrm{M} \alpha)$ was measured on LPET and the peak counting time was 300 seconds with background measured on both sides at half the peak time. For uranium, UM $\alpha$ line was used in order to avoid the interference of ThM $\beta$ line. In order to counter the loss of count rate, a peak counting time of 200 seconds (and 200 seconds for background by counting on both sides of the peak) was used for $\mathrm{Um} \beta$ line. $\mathrm{U}$ and Th were measured on PET. Thorium M $\beta$ peak was also counted for 200 seconds. Rare Earth elements measured included $\mathrm{La}(\mathrm{L} \alpha), \mathrm{Ce}(\mathrm{L} \alpha), \mathrm{Nd}(\mathrm{L} \beta), \operatorname{Pr}(\mathrm{L} \alpha), \mathrm{Sm}$ $(\operatorname{L} \alpha), \operatorname{Ho}(\mathrm{L} \beta)$, Dy $(\mathrm{L} \alpha)$ and $\mathrm{Gd}(\mathrm{L} \alpha)$. Counting time at peak varied between 40-60 seconds for these elements. Y L $\alpha$ line was used for yttrium and it was counted for 40 seconds on peak. No solution for correcting interference of $\mathrm{Y} \mathrm{L} \gamma$ on $\mathrm{PbM} \alpha$ could be arrived at using Virtual WDS. The overlap correction procedure for this interference was used. The raw data was corrected using PAP (Pouchoir and Pichoir, 1991) correction method which is inbuilt in software.

Standards used for $\mathrm{Pb}, \mathrm{U}$ and $\mathrm{Th}$ were $\mathrm{Pb}_{5}\left(\mathrm{PO}_{4}\right)_{3} \mathrm{Cl}, \mathrm{UO}_{2}$ and $\mathrm{ThO}_{2}$ and synthetic silica-aluminium glass containing $4 \%$ rare earth elements were used as standards for La, Ce, Nd, Pr, Sm, Ho, Dy and Gd. Phosphorus was calibrated with apatite. For yttrium aluminium garnet (YAG) was used. $\mathrm{Al}$ and Fe were calibrated using $\mathrm{Al}_{2} \mathrm{O}_{3}$ and $\mathrm{Fe}_{2} \mathrm{O}_{3}$ while for silica orthoclase was used. Further details of the analytical procedure are given in Pant et al. (2009). Complete analyses of monazite were obtained for chemical dating and the results with $\mathrm{Si}+\mathrm{P}+\mathrm{S}$ value at $\sim 1.0$ (normalization of cations at $4 \mathrm{O}$ basis) were used for further processing. For individual spot ages, the formulation of Montel et al. (1996) was followed, whereas the age probability plots and unmixing of ages were obtained using the software Isoplot3 (v.3.71.09.06.19nx) (Ludwig, 2003). Uncertainties in individual analyses in the data table and in weighted mean ages are quoted at the $95 \%$ confidence level $(2 \sigma)$. Electron Microprobe analyses of monazites along with their calculated spot ages are given in Table 1.

\section{Monazite chemistry}

\section{Regional metamorphic grains (sample GG-5)}

Six monazite grains in the sample were examined out of which grain 1 is present as inclusion in staurolite and grains 2, 3, 4, 5 and 6 are present in matrix (Fig. 6). Grain1 is elongated, euhedral with maximum dimension of $\sim 60 \mu \mathrm{m}$. Grain2 is subhedral with the length $15 \mu \mathrm{m}$ while grain 3 has length of around $50 \mu \mathrm{m}$. Both of these matrix grains are deeply embayed. Grain4 is euhedral and rounded with diameter $30 \mu \mathrm{m}$ and grain5, which is of similar size is anhedral. Grain 6 is relatively large in size and subhedral (not shown in Fig. 6).

$\mathrm{X}$-ray elemental mapping of $\mathrm{U}, \mathrm{Th}, \mathrm{Ce}$ and $\mathrm{Y}$ of two matrix monazite grains (grain 4 and grain5) are shown in Fig. 7 and 8. Black to blue colour in these maps indicates absence of the element while red colour shows higher content of the mapped element. The Ce Xray maps show that the two matrix monazites are Ce- monazites (Fig. 7a, 8a). Grain 4 is euhedral while grain 5 is anhedral and resorbed. High Th and high Y fragments in this grain (right central part of the $\mathrm{X}$-ray maps; Fig. 8) indicate that these possibly represent remaining parts of a larger grain. The core of the grain 4 is Th deficient while rim is Th-rich (Fig. 7b). Similar pattern is visible in grain5 although not with the same prominence. These grains show zoning of Th from core to rim but boundary of zone is irregular. Both grains are $\mathrm{U}$ poor and $\mathrm{Y}$ rich monazites (Fig.7 c,d and $8 \mathrm{c}, \mathrm{d}$ ).

The inclusion and the matrix monazite grains show large compositional variation. Inclusion monazite is depleted in REE as compared to matrix grains while it has higher $\mathrm{Th}+\mathrm{U}$ than matrix grains (Fig. 9). Grain1 is also depleted in LREE as compared to matrix grains and show higher huttonite content (Fig. 10). All six grains have almost similar $\mathrm{Y}$ content but $\mathrm{Th} / \mathrm{U}$ ratio is significantly higher in grain 1 (Fig. 11). Variation between brabantite and huttonite shows that grain 1 has positive brabantite and huttonite component while other grains only have huttonite component (Fig. 12).

In view of two sets of chemically distinct monazites the age data was analyzed in two groups. The spot ages of six grains are shown in Fig. 13. Grain1 represents dominantly one age population and the probability density plot of this grain gives an age of $917 \pm 19 \mathrm{Ma}$ 


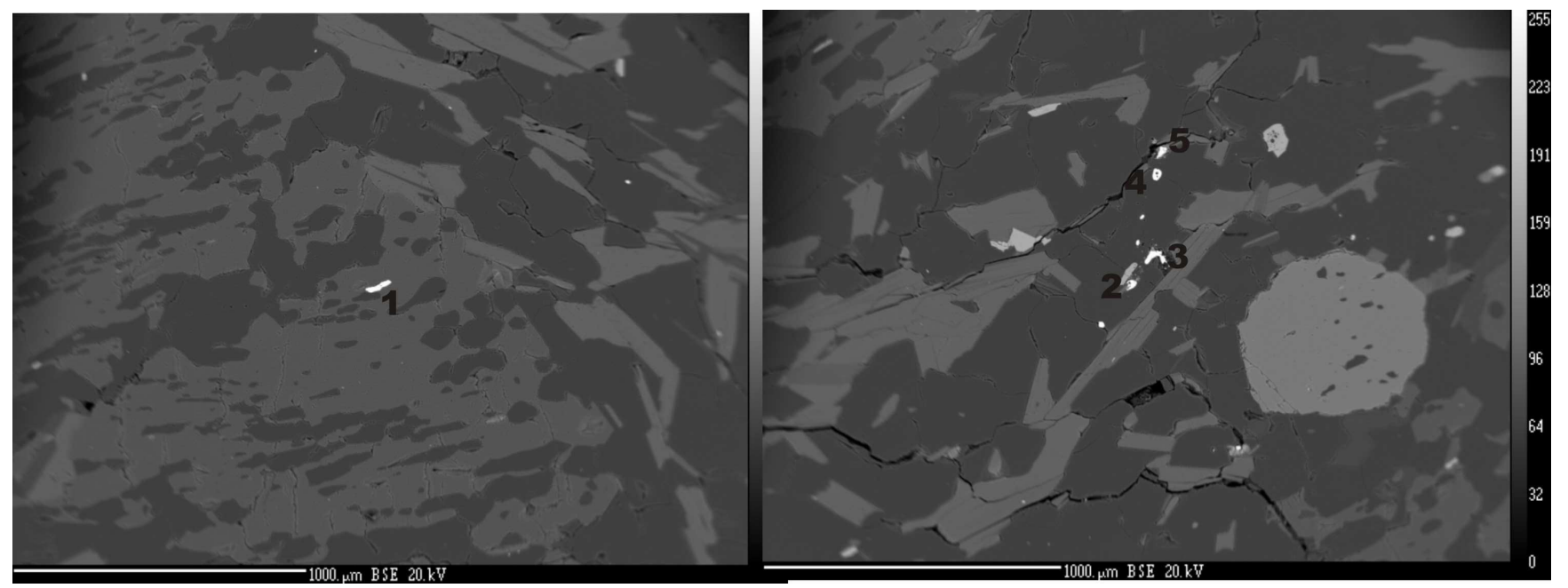

Fig.6. BSE images of inclusion and matrix monazite grains in garnet staurolite schist. Grain1 is present as inclusion in staurolite and grains $2,3,4,5$ and 6 are present in matrix.

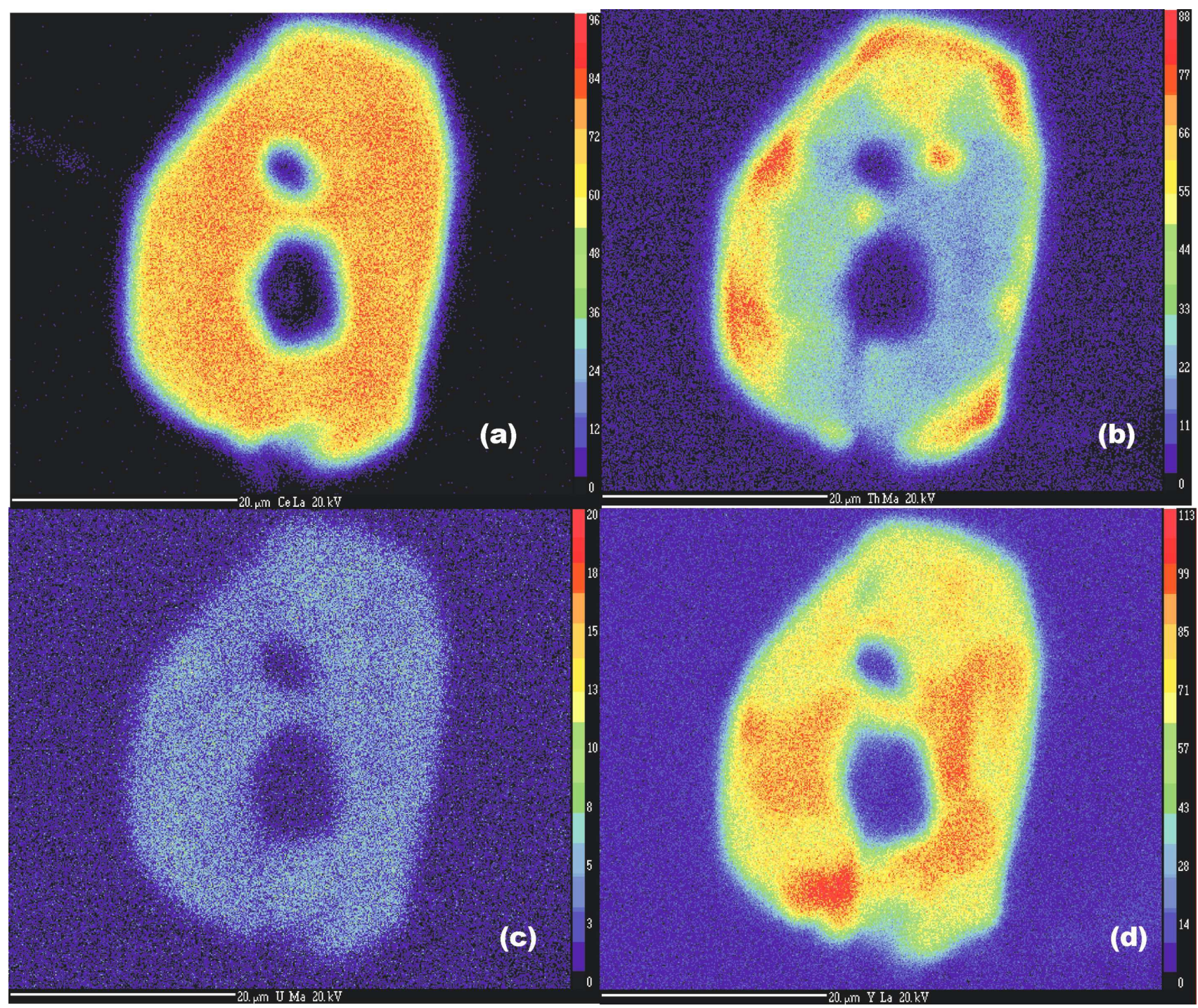

Fig7. X-ray elemental maps of Ce (a), Th (b), $U(c)$, and $Y(d)$ of grain 4. 


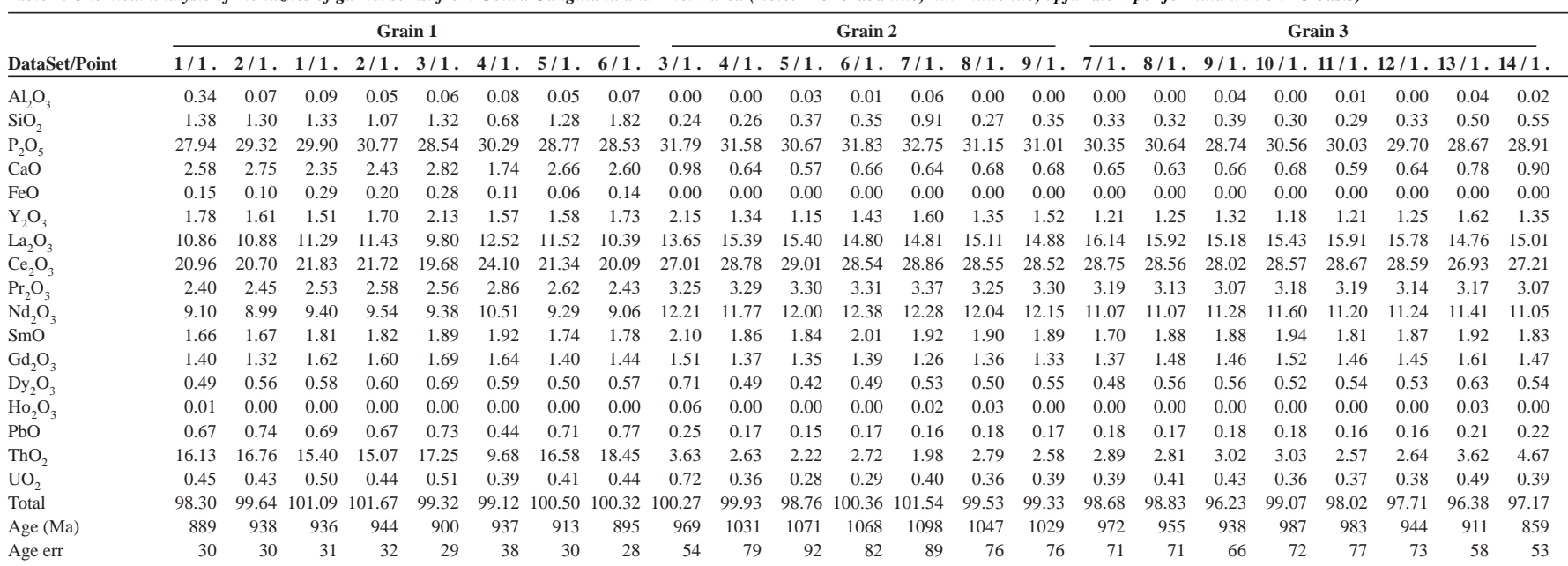
$\begin{array}{llllllllllllllllllllllll}4(\mathrm{O}) & 4(\mathrm{O}) & 4(\mathrm{O}) & 4(\mathrm{O}) & 4(\mathrm{O}) & 4(\mathrm{O}) & 4(\mathrm{O}) & 4(\mathrm{O}) & 4(\mathrm{O}) & 4(\mathrm{O}) & 4(\mathrm{O}) & 4(\mathrm{O}) & 4(\mathrm{O}) & 4(\mathrm{O}) & 4(\mathrm{O}) & 4(\mathrm{O}) & 4(\mathrm{O}) & 4(\mathrm{O}) & 4(\mathrm{O}) & 4(\mathrm{O}) & 4(\mathrm{O}) & 4(\mathrm{O}) & 4(\mathrm{O}) \\ 0.11 & 0.115 & 0.097 & 0.099 & 0.120 & 0.073 & 0.112 & 0.110 & 0.040 & 0.026 & 0.024 & 0.027 & 0.025 & 0.028 & 0.028 & 0.027 & 0.026 & 0.029 & 0.029 & 0.025 & 0.027 & 0.034 & 0.039\end{array}$

Formula $\begin{array}{llllllllllllllllllllllll}0.005 & 0.003 & 0.009 & 0.006 & 0.009 & 0.004 & 0.002 & 0.005 & 0.000 & 0.000 & 0.000 & 0.000 & 0.000 & 0.000 & 0.000 & 0.000 & 0.000 & 0.000 & 0.000 & 0.000 & 0.000 & 0.000 & 0.000\end{array}$ $\begin{array}{llllllllllllllllllllllll}0.024 & 0.024 & 0.025 & 0.025 & 0.027 & 0.027 & 0.025 & 0.025 & 0.029 & 0.026 & 0.026 & 0.028 & 0.026 & 0.027 & 0.026 & 0.024 & 0.026 & 0.028 & 0.027 & 0.026 & 0.027 & 0.028 & 0.027\end{array}$ $\begin{array}{llllllllllllllllllllllll}0.007 & 0.008 & 0.007 & 0.007 & 0.008 & 0.005 & 0.007 & 0.008 & 0.003 & 0.002 & 0.002 & 0.002 & 0.002 & 0.002 & 0.002 & 0.002 & 0.002 & 0.002 & 0.002 & 0.002 & 0.002 & 0.002 & 0.002\end{array}$

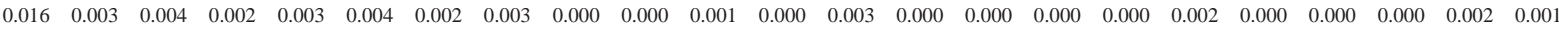

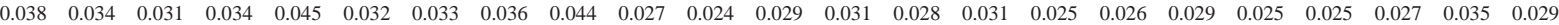
$\begin{array}{lllllllllllllllllllllll}0.161 & 0.157 & 0.160 & 0.160 & 0.143 & 0.180 & 0.167 & 0.151 & 0.191 & 0.217 & 0.221 & 0.207 & 0.202 & 0.215 & 0.212 & 0.233 & 0.229 & 0.228 & 0.222 & 0.232 & 0.232 & 0.221 & 0.223\end{array}$ $\begin{array}{llllllllllllllllllllllll}0.308 & 0.297 & 0.308 & 0.302 & 0.286 & 0.344 & 0.307 & 0.289 & 0.376 & 0.403 & 0.414 & 0.397 & 0.390 & 0.403 & 0.404 & 0.413 & 0.408 & 0.418 & 0.408 & 0.415 & 0.417 & 0.401 & 0.402\end{array}$ $\begin{array}{lllllllllllllllllllllll}0.035 & 0.035 & 0.035 & 0.036 & 0.037 & 0.041 & 0.038 & 0.035 & 0.045 & 0.046 & 0.047 & 0.046 & 0.045 & 0.046 & 0.046 & 0.046 & 0.044 & 0.046 & 0.045 & 0.046 & 0.046 & 0.047 & 0.045 \\ 0.130 & 0.126 & 0.29 & 0.129 & 0.133 & 0.16 & 0.31 & 0.127 & 0.166 & 0.16 & 0.67 & 0.168 & 0.162 & 0.166 & 0.68 & 0.155 & 0.154 & 0.164 & 0.62 & 0.158 & 0.160 & 0.166 & 0.159\end{array}$

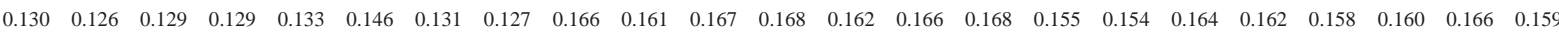
$\begin{array}{lllllllllllllllllllllll}0.019 & 0.017 & 0.021 & 0.020 & 0.022 & 0.021 & 0.018 & 0.019 & 0.019 & 0.017 & 0.017 & 0.017 & 0.015 & 0.017 & 0.017 & 0.018 & 0.019 & 0.020 & 0.020 & 0.019 & 0.019 & 0.022 & 0.020\end{array}$ $\begin{array}{llllllllllllllllllllllll}0.006 & 0.007 & 0.007 & 0.007 & 0.009 & 0.007 & 0.006 & 0.007 & 0.009 & 0.006 & 0.005 & 0.006 & 0.006 & 0.006 & 0.007 & 0.006 & 0.007 & 0.007 & 0.007 & 0.007 & 0.007 & 0.008 & 0.007\end{array}$ $\begin{array}{llllllllllllllllllllllll}0.000 & 0.000 & 0.000 & 0.000 & 0.000 & 0.000 & 0.000 & 0.000 & 0.001 & 0.000 & 0.000 & 0.000 & 0.000 & 0.000 & 0.000 & 0.000 & 0.000 & 0.000 & 0.000 & 0.000 & 0.000 & 0.000 & 0.000\end{array}$

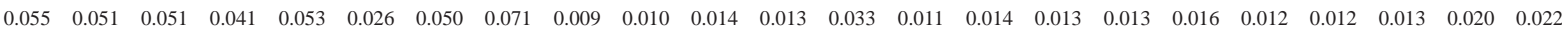
$\begin{array}{lllllllllllllllllllllllll}0.147 & 0.149 & 0.135 & 0.130 & 0.156 & 0.086 & 0.148 & 0.165 & 0.031 & 0.023 & 0.020 & 0.024 & 0.017 & 0.024 & 0.023 & 0.026 & 0.025 & 0.028 & 0.027 & 0.023 & 0.024 & 0.034 & 0.043 \\ 0.004 & 0.004 & 0.04 & 0.004 & 0.005 & 0.003 & 0.04 & 0.004 & 0.006 & 0.003 & 0.02 & 0.002 & 0.003 & 0.003 & 0.03 & 0.003 & 0.004 & 0.004 & 0.003 & 0.003 & 0.003 & 0.004 & 0.003\end{array}$ $\begin{array}{llllllllllllllllllllllllll}0.004 & 0.004 & 0.004 & 0.004 & 0.005 & 0.003 & 0.004 & 0.004 & 0.006 & 0.003 & 0.002 & 0.002 & 0.003 & 0.003 & 0.003 & 0.003 & 0.004 & 0.004 & 0.003 & 0.003 & 0.003 & 0.004 & 0.003\end{array}$ $\begin{array}{llllllllllllllllllllllll}0.948 & 0.972 & 0.975 & 0.990 & 0.958 & 0.999 & 0.958 & 0.949 & 1.024 & 1.023 & 1.012 & 1.024 & 1.023 & 1.018 & 1.015 & 1.007 & 1.012 & 0.991 & 1.010 & 1.006 & 1.001 & 0.987 & 0.987\end{array}$

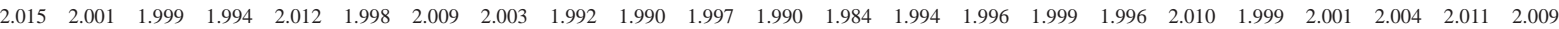
Total

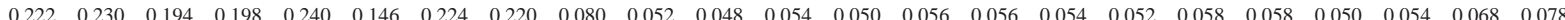

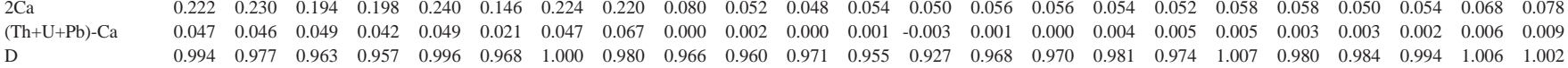
$\mathrm{D}$

$\mathrm{XBrb}$

Xhut

XLREE

XHREE

$\mathrm{XYPO}$

$\mathrm{Th} / \mathrm{U}$

Th (ppb)

$\mathrm{U}(\mathrm{ppb})$

$\mathrm{Ce} / \mathrm{Sm}$

$\mathrm{Ca}+\mathrm{Si} /(\mathrm{Th}+\mathrm{U}+\mathrm{Pb})$

$\mathrm{REE}+\mathrm{P}+\mathrm{Y}$

$\mathrm{Th}+\mathrm{U}+\mathrm{S}$

$\begin{array}{lllllllllllllllllllllll}0.223 & 0.235 & 0.201 & 0.207 & 0.241 & 0.151 & 0.224 & 0.224 & 0.083 & 0.054 & 0.049 & 0.057 & 0.054 & 0.058 & 0.058 & 0.055 & 0.053 & 0.058 & 0.059 & 0.051 & 0.054 & 0.068 & 0.078\end{array}$ $\begin{array}{lllllllllllllllllllllll}0.047 & 0.047 & 0.051 & 0.044 & 0.049 & 0.022 & 0.047 & 0.068 & 0.000 & 0.002 & 0.000 & 0.001 & -0.003 & 0.001 & 0.000 & 0.004 & 0.005 & 0.005 & 0.003 & 0.003 & 0.002 & 0.006 & 0.009\end{array}$

$\begin{array}{llllllllllllllllllllllll}0.658 & 0.639 & 0.657 & 0.652 & 0.626 & 0.738 & 0.668 & 0.627 & 0.807 & 0.853 & 0.875 & 0.846 & 0.825 & 0.857 & 0.856 & 0.871 & 0.861 & 0.884 & 0.864 & 0.877 & 0.882 & 0.863 & 0.856\end{array}$ $\begin{array}{lllllllllllllllllllllll}0.025 & 0.024 & 0.028 & 0.027 & 0.031 & 0.028 & 0.024 & 0.026 & 0.029 & 0.023 & 0.022 & 0.023 & 0.021 & 0.023 & 0.024 & 0.024 & 0.026 & 0.027 & 0.027 & 0.026 & 0.026 & 0.030 & 0.027\end{array}$ $\begin{array}{lllllllllllllllllllllll}0.131 & 0.129 & 0.134 & 0.135 & 0.134 & 0.151 & 0.131 & 0.130 & 0.172 & 0.168 & 0.172 & 0.176 & 0.175 & 0.171 & 0.173 & 0.158 & 0.158 & 0.163 & 0.165 & 0.161 & 0.161 & 0.165 & 0.159\end{array}$

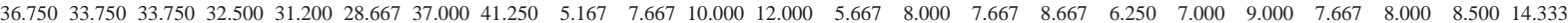

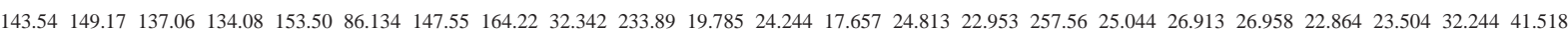
$\begin{array}{lllllllllllllllllllllll}3.942 & 3.810 & 4.364 & 3.828 & 4.505 & 3.458 & 3.616 & 3.854 & 6.353 & 3.168 & 2.490 & 2.534 & 3.546 & 3.194 & 3.458 & 3.405 & 3.599 & 3.766 & 3.159 & 3.229 & 3.379 & 4.312 & 3.405\end{array}$

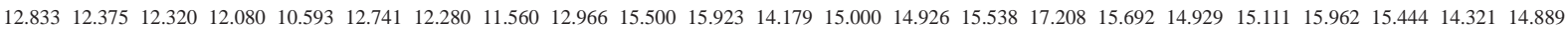

$\begin{array}{lllllllllllllllllllllll}0.151 & 0.148 & 0.133 & 0.125 & 0.155 & 0.091 & 0.146 & 0.162 & 0.046 & 0.034 & 0.037 & 0.038 & 0.056 & 0.037 & 0.040 & 0.039 & 0.037 & 0.044 & 0.039 & 0.036 & 0.039 & 0.053 & 0.059\end{array}$

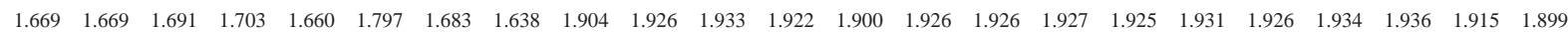

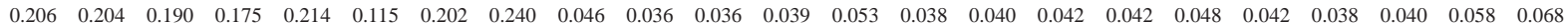

$\mathrm{Y}(\mathrm{ppm})$

$\mathrm{Si}+\mathrm{P}$

U+th+LREE+

HREE

$\mathrm{Ca}+\mathrm{Si}$

$\mathrm{U}+\mathrm{Th}+\mathrm{Pb}$

$\mathrm{La}^{*}$
Y+HREE

$\mathrm{Ca}+\mathrm{Th}+\mathrm{U}$

$2 \mathrm{ThSiO}_{4}$

$2 \mathrm{REEPO}_{4}$

$\mathrm{CaTh}(\mathrm{PO})_{2}$

$\mathrm{P}+\mathrm{Y}+\mathrm{REE}$ (apfu) $\mathrm{Th}+\mathrm{U}+\mathrm{Si}$ (apfu)

$(\mathrm{Ce}+\mathrm{La}+\mathrm{Nd}) / \mathrm{Th}$ $\mathrm{Ce} / \mathrm{Th}$

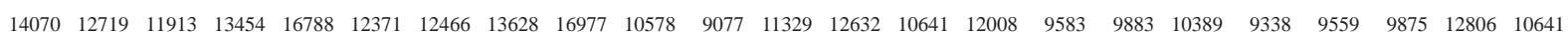
$\begin{array}{lllllllllllllllllllllll}1.003 & 1.023 & 1.026 & 1.031 & 1.011 & 1.025 & 1.008 & 1.020 & 1.033 & 1.033 & 1.026 & 1.037 & 1.056 & 1.029 & 1.029 & 1.020 & 1.025 & 1.007 & 1.022 & 1.018 & 1.014 & 1.007 & 1.009\end{array}$ $\begin{array}{lllllllllllllllllllllll}0.834 & 0.816 & 0.824 & 0.813 & 0.818 & 0.855 & 0.844 & 0.822 & 0.873 & 0.902 & 0.919 & 0.895 & 0.866 & 0.907 & 0.906 & 0.924 & 0.916 & 0.943 & 0.921 & 0.929 & 0.935 & 0.931 & 0.929\end{array}$

$\begin{array}{lllllllllllllllllllllll}0.166 & 0.166 & 0.148 & 0.140 & 0.173 & 0.099 & 0.162 & 0.181 & 0.049 & 0.036 & 0.038 & 0.040 & 0.058 & 0.039 & 0.042 & 0.040 & 0.039 & 0.045 & 0.041 & 0.037 & 0.040 & 0.054 & 0.061\end{array}$ $\begin{array}{lllllllllllllllllllllllllllll}0.158 & 0.161 & 0.146 & 0.141 & 0.169 & 0.094 & 0.159 & 0.177 & 0.040 & 0.028 & 0.024 & 0.028 & 0.022 & 0.029 & 0.028 & 0.031 & 0.031 & 0.034 & 0.032 & 0.028 & 0.029 & 0.040 & 0.048\end{array}$

$\begin{array}{lllllllllllllllllllllll}0.658 & 0.639 & 0.657 & 0.652 & 0.626 & 0.738 & 0.668 & 0.627 & 0.807 & 0.853 & 0.875 & 0.846 & 0.825 & 0.857 & 0.856 & 0.871 & 0.861 & 0.884 & 0.864 & 0.877 & 0.882 & 0.863 & 0.856\end{array}$ $\begin{array}{lllllllllllllllllllllllll}0.063 & 0.058 & 0.059 & 0.061 & 0.076 & 0.060 & 0.057 & 0.062 & 0.073 & 0.050 & 0.046 & 0.052 & 0.052 & 0.051 & 0.055 & 0.049 & 0.052 & 0.056 & 0.052 & 0.051 & 0.053 & 0.065 & 0.056\end{array}$ $\begin{array}{lllllllllllllllllllllllllll}0.262 & 0.268 & 0.236 & 0.233 & 0.281 & 0.162 & 0.264 & 0.279 & 0.077 & 0.052 & 0.046 & 0.053 & 0.045 & 0.055 & 0.054 & 0.056 & 0.055 & 0.061 & 0.059 & 0.051 & 0.054 & 0.072 & 0.085\end{array}$

$\begin{array}{lllllllllllllllllllllll}0.404 & 0.400 & 0.372 & 0.342 & 0.418 & 0.224 & 0.396 & 0.472 & 0.080 & 0.066 & 0.068 & 0.074 & 0.100 & 0.070 & 0.074 & 0.078 & 0.076 & 0.088 & 0.078 & 0.070 & 0.074 & 0.108 & 0.130\end{array}$

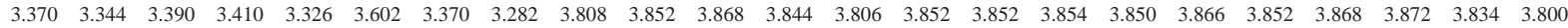

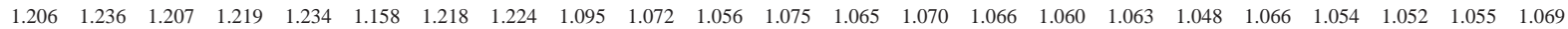

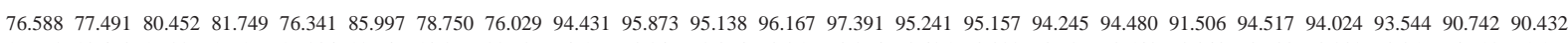

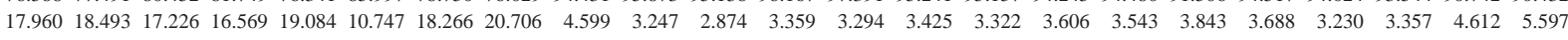

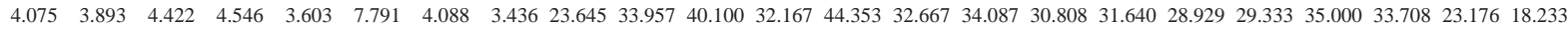
$\begin{array}{llllllllllllllllllllllllllll}2.095 & 1.993 & 2.281 & 2.323 & 1.833 & 4.000 & 2.074 & 1.752 & 12.129 & 17.522 & 20.700 & 16.542 & 22.941 & 16.792 & 17.565 & 15.885 & 16.320 & 14.929 & 15.111 & 18.043 & 17.375 & 11.794 & 9.349\end{array}$ 
Table 1 Contd...

\begin{tabular}{|c|c|c|c|c|c|c|c|c|c|c|c|c|c|c|c|c|c|c|c|c|c|c|c|c|}
\hline \multirow[b]{2}{*}{ DataSet/Point } & \multicolumn{8}{|c|}{ Grain 4} & \multicolumn{6}{|c|}{ Grain 5} & \multicolumn{10}{|c|}{ Grain 6} \\
\hline & & & & & & & & & & & 7. & 07 & & & 11 & & & & $3 / 1$ & $34 /$ & 87 & 07 & 7 & $8 / 1$ \\
\hline $\mathrm{Al}_{2} \mathrm{O}_{3}$ & 0.04 & 0.01 & 0.05 & 0.01 & 0.00 & 0.00 & 0.00 & 0.03 & 0.01 & 0.01 & 0.01 & 0.00 & 0.10 & 0.01 & 0.01 & 0.01 & 0.00 & 0.00 & 0.00 & 0.00 & 0.01 & 0.01 & 0.03 & 0.00 \\
\hline $\mathrm{SiO}_{2}$ & 0.40 & 0.25 & 0.23 & 0.34 & 0.42 & 0.22 & 0.26 & 0.25 & 0.25 & 0.21 & 0.27 & 0.20 & 0.43 & 0.21 & 0.25 & 0.25 & 0.22 & 0.22 & 0.24 & 0.20 & 0.26 & 0.20 & 0.22 & 0.20 \\
\hline $\mathrm{P}_{2} \mathrm{O}_{5}$ & 30.64 & 30.97 & 31.30 & 30.98 & 31.04 & 31.14 & 30.94 & 30.10 & 31.32 & 31.54 & 32.20 & 31.43 & 31.51 & 30.93 & 31.51 & 30.69 & 31.18 & 30.75 & 30.87 & 30.86 & 30.74 & 30.97 & 30.68 & 31.28 \\
\hline $\mathrm{CaO}$ & 0.56 & 0.46 & 0.49 & 0.51 & 0.55 & 0.37 & 0.32 & 0.28 & 0.45 & 0.55 & 1.12 & 0.59 & 1.03 & 0.74 & 0.37 & 0.36 & 0.41 & 0.42 & 0.40 & 0.47 & 0.47 & 0.32 & 0.50 & 0.41 \\
\hline $\mathrm{FeO}$ & 0.00 & 0.00 & 0.00 & 0.00 & 0.00 & 0.00 & 0.00 & 0.00 & 0.00 & 0.00 & 0.00 & 0.00 & 0.02 & 0.00 & 0.00 & 0.00 & 0.00 & 0.00 & 0.00 & 0.00 & 0.00 & 0.00 & 0.00 & 0.00 \\
\hline $\mathrm{Y}_{2} \mathrm{O}_{3}$ & 1.36 & 1.79 & 1.74 & 1.18 & 1.37 & 1.34 & 1.76 & 1.78 & 1.17 & 1.13 & 1.10 & 1.42 & 1.07 & 1.52 & 1.35 & 1.16 & 1.53 & 1.49 & 1.42 & 1.67 & 1.28 & 1.15 & 1.23 & 1.53 \\
\hline $\mathrm{La}_{2} \mathrm{O}_{3}$ & 16.25 & 17.01 & 17.04 & 16.73 & 16.02 & 17.14 & 17.34 & 17.40 & 18.26 & 18.74 & 16.54 & 18.09 & 17.43 & 17.45 & 17.16 & 17.45 & 16.85 & 16.76 & 16.92 & 16.50 & 16.78 & 17.07 & 16.75 & 16.66 \\
\hline $\mathrm{Ce}_{2} \mathrm{O}_{3}$ & 28.78 & 29.50 & 29.52 & 29.03 & 28.76 & 29.79 & 29.81 & 29.83 & 30.69 & 30.70 & 29.37 & 29.88 & 29.88 & 29.62 & 29.78 & 30.03 & 29.46 & 29.34 & 29.49 & 29.21 & 29.41 & 30.10 & 29.41 & 29.54 \\
\hline $\mathrm{Pr}_{2} \mathrm{O}_{3}$ & 3.12 & 3.09 & 3.08 & 3.11 & 3.18 & 3.23 & 3.17 & 3.07 & 3.11 & 3.11 & 3.13 & 3.08 & 3.07 & 3.13 & 3.13 & 3.22 & 3.19 & 3.22 & 3.20 & 3.22 & 3.19 & 3.26 & 3.17 & 3.22 \\
\hline $\mathrm{Nd}_{2} \mathrm{O}_{3}$ & 10.88 & 10.53 & 10.53 & 10.84 & 11.05 & 10.75 & 10.50 & 10.39 & 10.32 & 10.08 & 10.94 & 10.05 & 10.34 & 10.38 & 11.02 & 10.83 & 11.11 & 11.15 & 10.97 & 11.13 & 11.00 & 11.21 & 10.98 & 11.25 \\
\hline $\mathrm{SmO}$ & 1.65 & 1.49 & 1.47 & 1.68 & 1.78 & 1.56 & 1.46 & 1.49 & 1.35 & 1.28 & 1.69 & 1.37 & 1.46 & 1.48 & 1.55 & 1.51 & 1.59 & 1.66 & 1.56 & 1.63 & 1.57 & 1.54 & 1.54 & 1.66 \\
\hline $\mathrm{Gd}_{2} \mathrm{O}_{3}$ & 1.28 & 1.11 & 1.07 & 1.24 & 1.36 & 1.09 & 1.07 & 0.96 & 0.85 & 0.80 & 1.27 & 0.86 & 0.91 & 1.04 & 1.09 & 0.92 & 1.17 & 1.22 & 1.17 & 1.17 & 1.09 & 1.07 & 0.98 & 1.21 \\
\hline $\mathrm{Dy}_{2} \mathrm{O}_{3}$ & 0.46 & 0.50 & 0.53 & 0.45 & 0.50 & 0.46 & 0.52 & 0.52 & 0.33 & 0.34 & 0.43 & 0.42 & 0.38 & 0.44 & 0.47 & 0.40 & 0.49 & 0.51 & 0.47 & 0.52 & 0.41 & 0.39 & 0.41 & 0.51 \\
\hline $\mathrm{Ho}_{2} \mathrm{O}_{3}$ & 0.01 & 0.03 & 0.01 & 0.00 & 0.00 & 0.00 & 0.05 & 0.00 & 0.00 & 0.00 & 0.01 & 0.01 & 0.00 & 0.04 & 0.00 & 0.00 & 0.02 & 0.03 & 0.00 & 0.04 & 0.00 & 0.00 & 0.00 & 0.00 \\
\hline $\mathrm{PbO}$ & 0.18 & 0.12 & 0.13 & 0.18 & 0.17 & 0.13 & 0.10 & 0.10 & 0.08 & 0.08 & 0.17 & 0.13 & 0.11 & 0.12 & 0.12 & 0.11 & 0.12 & 0.13 & 0.13 & 0.13 & 0.15 & 0.11 & 0.16 & 0.11 \\
\hline $\mathrm{ThO}_{2}$ & 2.68 & 1.13 & 1.24 & 2.42 & 2.57 & 1.55 & 0.75 & 0.69 & 0.56 & 0.46 & 2.41 & 1.01 & 1.42 & 1.19 & 1.87 & 1.78 & 2.00 & 2.12 & 2.05 & 2.00 & 2.57 & 1.73 & 2.62 & 1.59 \\
\hline $\mathrm{UO}_{2}{ }^{2}$ & 0.32 & 0.33 & 0.36 & 0.36 & 0.34 & 0.28 & 0.36 & 0.37 & 0.18 & 0.19 & 0.35 & 0.45 & 0.25 & 0.33 & 0.16 & 0.13 & 0.16 & 0.17 & 0.16 & 0.24 & 0.14 & 0.11 & 0.23 & 0.16 \\
\hline Total & 98.59 & 98.30 & 98.77 & 99.04 & 99.12 & 99.05 & 98.42 & 97.28 & 98.90 & 99.21 & 101.00 & 98.98 & 99.37 & 98.61 & 99.82 & 98.85 & 99.49 & 99.18 & 99.02 & 98.98 & 99.06 & 99.23 & 98.89 & 99.34 \\
\hline Age (Ma) & 1107 & 1176 & 1180 & 1113 & 1067 & 1143 & 1204 & 1201 & 1506 & 1627 & 1077 & 1179 & 1101 & 1184 & 1113 & 1173 & 1086 & 1094 & 1131 & 1042 & 1133 & 1171 & 1059 & 1139 \\
\hline Age err & 82 & 118 & 112 & 84 & 81 & 112 & 131 & 131 & 233 & 248 & 85 & 110 & 122 & 119 & 117 & 128 & 109 & 105 & 111 & 99 & 98 & 134 & 88 & 127 \\
\hline
\end{tabular}

$\begin{array}{llllllllllllllllllllllllll}\text { Formula } & 4(\mathrm{O}) & 4(\mathrm{O}) & 4(\mathrm{O}) & 4(\mathrm{O}) & 4(\mathrm{O}) & 4(\mathrm{O}) & 4(\mathrm{O}) & 4(\mathrm{O}) & 4(\mathrm{O}) & 4(\mathrm{O}) & 4(\mathrm{O}) & 4(\mathrm{O}) & 4(\mathrm{O}) & 4(\mathrm{O}) & 4(\mathrm{O}) & 4(\mathrm{O}) & 4(\mathrm{O}) & 4(\mathrm{O}) & 4(\mathrm{O}) & 4(\mathrm{O}) & 4(\mathrm{O}) & 4(\mathrm{O}) & 4(\mathrm{O}) & 4(\mathrm{O})\end{array}$

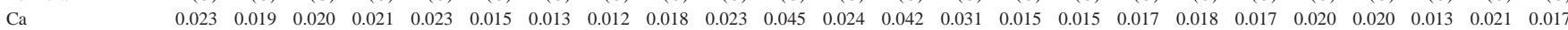
$\begin{array}{lllllllllllllllllllllllllllll}\mathrm{Ca} & \mathrm{Fe} & 0.000 & 0.000 & 0.000 & 0.000 & 0.000 & 0.000 & 0.000 & 0.000 & 0.000 & 0.000 & 0.000 & 0.000 & 0.001 & 0.000 & 0.000 & 0.000 & 0.000 & 0.000 & 0.000 & 0.000 & 0.000 & 0.000 & 0.000 & 0.000\end{array}$

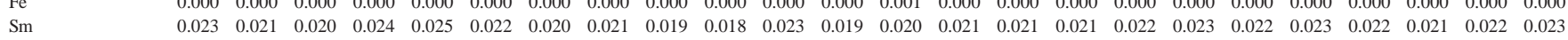

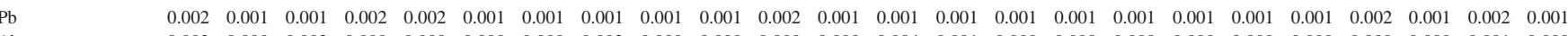

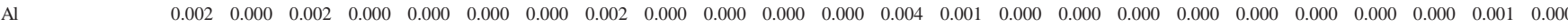
$\begin{array}{llllllllllllllllllllllllllll}\mathrm{Y} & 0.028 & 0.037 & 0.036 & 0.024 & 0.028 & 0.028 & 0.036 & 0.038 & 0.024 & 0.023 & 0.022 & 0.029 & 0.022 & 0.031 & 0.027 & 0.024 & 0.031 & 0.031 & 0.029 & 0.035 & 0.026 & 0.024 & 0.025 & 0.031\end{array}$ $\begin{array}{lllllllllllllllllllllllll}\mathrm{La} & 0.234 & 0.244 & 0.242 & 0.239 & 0.228 & 0.245 & 0.249 & 0.254 & 0.259 & 0.265 & 0.229 & 0.257 & 0.245 & 0.250 & 0.242 & 0.251 & 0.240 & 0.240 & 0.242 & 0.236 & 0.241 & 0.244 & 0.241 & 0.237\end{array}$ $\begin{array}{llllllllllllllllllllllllll}\mathrm{Ce} & 0.411 & 0.420 & 0.417 & 0.412 & 0.407 & 0.422 & 0.424 & 0.432 & 0.433 & 0.431 & 0.404 & 0.421 & 0.417 & 0.421 & 0.418 & 0.429 & 0.416 & 0.418 & 0.419 & 0.416 & 0.419 & 0.427 & 0.420 & 0.417\end{array}$ $\begin{array}{lllllllllllllllllllllllllll}\text { Pr } & 0.044 & 0.044 & 0.043 & 0.044 & 0.045 & 0.046 & 0.045 & 0.044 & 0.044 & 0.043 & 0.043 & 0.043 & 0.043 & 0.044 & 0.044 & 0.046 & 0.045 & 0.046 & 0.045 & 0.046 & 0.045 & 0.046 & 0.045 & 0.045\end{array}$ $\begin{array}{llllllllllllllllllllllllllll}\mathrm{Nd} & 0.151 & 0.146 & 0.145 & 0.150 & 0.153 & 0.148 & 0.146 & 0.147 & 0.142 & 0.138 & 0.147 & 0.138 & 0.141 & 0.144 & 0.151 & 0.151 & 0.153 & 0.155 & 0.152 & 0.154 & 0.153 & 0.155 & 0.153 & 0.155\end{array}$

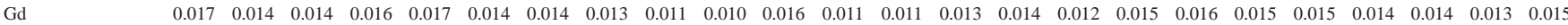
Dy $\quad \begin{array}{llllllllllllllllllllllllllllllll} & 0.006 & 0.006 & 0.007 & 0.006 & 0.006 & 0.006 & 0.006 & 0.007 & 0.004 & 0.004 & 0.005 & 0.005 & 0.005 & 0.005 & 0.006 & 0.005 & 0.006 & 0.006 & 0.006 & 0.007 & 0.005 & 0.005 & 0.005 & 0.006\end{array}$ $\begin{array}{llllllllllllllllllllllllll}\text { Ho } & 0.000 & 0.000 & 0.000 & 0.000 & 0.000 & 0.000 & 0.001 & 0.000 & 0.000 & 0.000 & 0.000 & 0.000 & 0.000 & 0.001 & 0.000 & 0.000 & 0.000 & 0.000 & 0.000 & 0.000 & 0.000 & 0.000 & 0.000 & 0.000\end{array}$ $\begin{array}{llllllllllllllllllllllllll}\mathrm{Si} & 0.015 & 0.010 & 0.009 & 0.013 & 0.016 & 0.009 & 0.010 & 0.010 & 0.009 & 0.008 & 0.010 & 0.008 & 0.016 & 0.008 & 0.009 & 0.010 & 0.008 & 0.009 & 0.009 & 0.008 & 0.010 & 0.008 & 0.009 & 0.008\end{array}$ $\begin{array}{llllllllllllllllllllllllllll}\text { Th } & 0.024 & 0.010 & 0.011 & 0.021 & 0.023 & 0.014 & 0.007 & 0.006 & 0.005 & 0.004 & 0.021 & 0.009 & 0.012 & 0.010 & 0.016 & 0.016 & 0.018 & 0.019 & 0.018 & 0.018 & 0.023 & 0.015 & 0.023 & 0.014\end{array}$ $\mathrm{U} \quad \quad \begin{array}{lllllllllllllllllllllllll}0.003 & 0.003 & 0.003 & 0.003 & 0.003 & 0.002 & 0.003 & 0.003 & 0.002 & 0.002 & 0.003 & 0.004 & 0.002 & 0.003 & 0.001 & 0.001 & 0.001 & 0.001 & 0.001 & 0.002 & 0.001 & 0.001 & 0.002 & 0.001\end{array}$ $\begin{array}{lllllllllllllllllllllllll}1.011 & 1.019 & 1.022 & 1.017 & 1.016 & 1.020 & 1.018 & 1.009 & 1.022 & 1.024 & 1.025 & 1.023 & 1.017 & 1.016 & 1.022 & 1.013 & 1.018 & 1.013 & 1.015 & 1.015 & 1.013 & 1.017 & 1.013 & 1.021\end{array}$

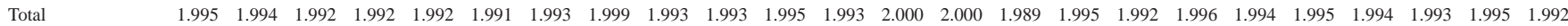

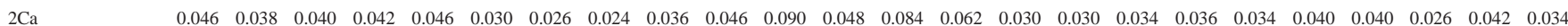

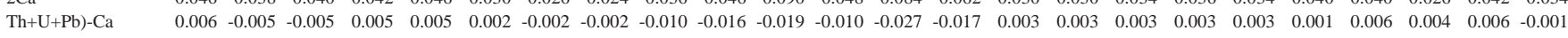
$\begin{array}{lllllllllllllllllllllllllll}\mathrm{D} & 0.969 & 0.968 & 0.962 & 0.965 & 0.963 & 0.965 & 0.968 & 0.981 & 0.964 & 0.964 & 0.963 & 0.965 & 0.963 & 0.978 & 0.957 & 0.973 & 0.966 & 0.975 & 0.968 & 0.975 & 0.972 & 0.967 & 0.974 & 0.963\end{array}$

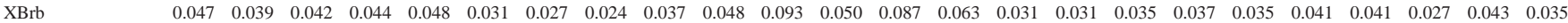
Xhut $\begin{array}{llllllllllllllllllllllllllll}0.006 & -0.005 & -0.005 & 0.005 & 0.005 & 0.002 & -0.002 & -0.002 & -0.010 & -0.017 & -0.020 & -0.010 & -0.028 & -0.017 & 0.003 & 0.003 & 0.003 & 0.003 & 0.003 & 0.001 & 0.006 & 0.004 & 0.006 & -0.001\end{array}$

XLREE

XHREE

$\mathrm{XYPO}_{4}$

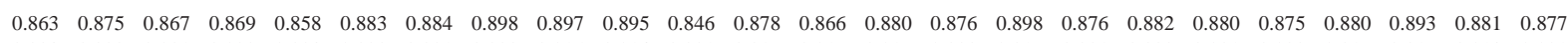

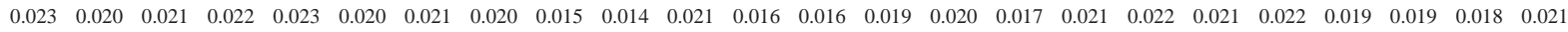
$\begin{array}{llllllllllllllllllllllll}0.156 & 0.151 & 0.151 & 0.155 & 0.159 & 0.153 & 0.151 & 0.150 & 0.147 & 0.143 & 0.153 & 0.143 & 0.146 & 0.147 & 0.158 & 0.155 & 0.158 & 0.159 & 0.157 & 0.158 & 0.157 & 0.160 & 0.157 & 0.161\end{array}$

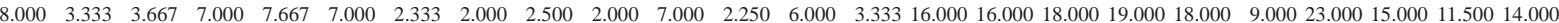

Th (ppb)

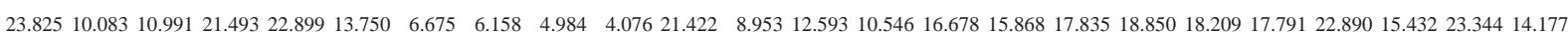

$\mathrm{U}(\mathrm{ppb})$

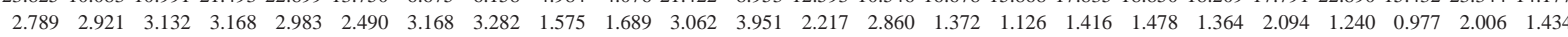

$\mathrm{Ce} / \mathrm{Sm}$

$17.87020 .00020 .85017 .167 \quad 16.28019 .18221 .20020 .57122 .78923 .944 \quad 17.56522 .15820 .85020 .04819 .90520 .42918 .90918 .17419 .04518 .08719 .04520 .333 \quad 19.091 \quad 18.130$

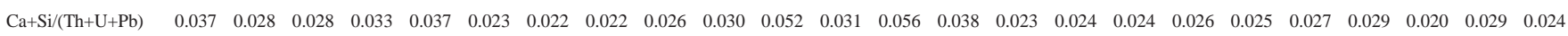

$\mathrm{REE}+\mathrm{P}+\mathrm{Y}$

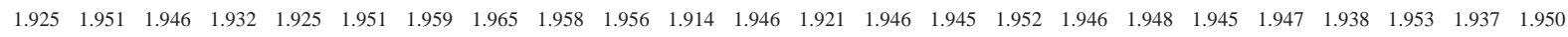

$\mathrm{Th}+\mathrm{U}+\mathrm{SI}$ $\begin{array}{llllllllllllllllllllllllll}0.042 & 0.023 & 0.023 & 0.037 & 0.042 & 0.025 & 0.020 & 0.019 & 0.016 & 0.014 & 0.034 & 0.021 & 0.030 & 0.021 & 0.026 & 0.027 & 0.027 & 0.029 & 0.028 & 0.028 & 0.034 & 0.024 & 0.034 & 0.023\end{array}$

$\mathrm{Y}(\mathrm{ppm})$

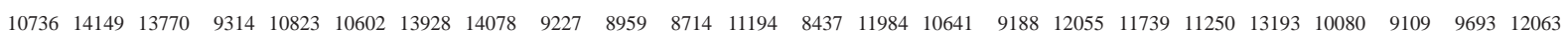

$\mathrm{Si}+\mathrm{P}$ $\begin{array}{lllllllllllllllllllllllll}1.026 & 1.029 & 1.031 & 1.030 & 1.032 & 1.029 & 1.028 & 1.019 & 1.031 & 1.032 & 1.035 & 1.031 & 1.033 & 1.024 & 1.031 & 1.023 & 1.026 & 1.022 & 1.024 & 1.023 & 1.023 & 1.025 & 1.022 & 1.029 & \end{array}$ $\begin{array}{lllllllllllllllllllllllllll}\text { U+th+LREE+ } & 0.913 & 0.908 & 0.902 & 0.915 & 0.907 & 0.919 & 0.915 & 0.927 & 0.919 & 0.915 & 0.891 & 0.907 & 0.896 & 0.912 & 0.913 & 0.932 & 0.916 & 0.924 & 0.920 & 0.917 & 0.923 & 0.928 & 0.924 & 0.913\end{array}$

HREE

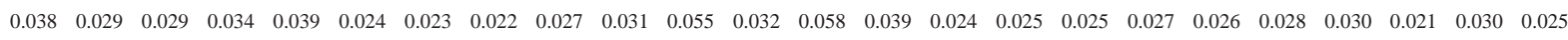

$\mathrm{Ca}+\mathrm{Si}$

$\mathrm{U}+\mathrm{Th}+\mathrm{Pb}$

La*
Y+HREE $\begin{array}{lllllllllllllllllllllllllll}0.029 & 0.014 & 0.015 & 0.026 & 0.028 & 0.017 & 0.011 & 0.010 & 0.008 & 0.007 & 0.026 & 0.014 & 0.015 & 0.014 & 0.018 & 0.018 & 0.020 & 0.021 & 0.020 & 0.021 & 0.026 & 0.017 & 0.027 & 0.016\end{array}$

$\mathrm{Ca}+\mathrm{Th}+\mathrm{U}$

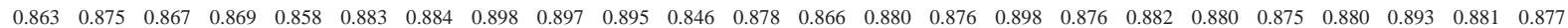
$\begin{array}{llllllllllllllllllllllllll}0.051 & 0.057 & 0.057 & 0.046 & 0.051 & 0.048 & 0.057 & 0.058 & 0.039 & 0.037 & 0.043 & 0.045 & 0.038 & 0.050 & 0.047 & 0.041 & 0.052 & 0.053 & 0.050 & 0.057 & 0.045 & 0.043 & 0.043 & 0.052\end{array}$ $\begin{array}{lllllllllllllllllllllllll}0.050 & 0.032 & 0.034 & 0.045 & 0.049 & 0.031 & 0.023 & 0.021 & 0.025 & 0.029 & 0.069 & 0.037 & 0.056 & 0.044 & 0.032 & 0.032 & 0.036 & 0.038 & 0.036 & 0.040 & 0.044 & 0.029 & 0.046 & 0.032\end{array}$

$2 \mathrm{ThSiO}_{4}$

$2 \mathrm{REEPO}_{4}$

$\begin{array}{llllllllllllllllllllllll}0.078 & 0.040 & 0.040 & 0.068 & 0.078 & 0.046 & 0.034 & 0.032 & 0.028 & 0.024 & 0.062 & 0.034 & 0.056 & 0.036 & 0.050 & 0.052 & 0.052 & 0.056 & 0.054 & 0.052 & 0.066 & 0.046 & 0.064 & 0.044 \\ 3.854 & 3.902 & 3.896 & 3.864 & 3.850 & 3.902 & 3.918 & 3.934 & 3.916 & 3.912 & 3.828 & 3.892 & 3.850 & 3.894 & 3.890 & 3.904 & 3.892 & 3.896 & 3.890 & 3.894 & 3.876 & 3.906 & 3.876 & 3.900\end{array}$ $\begin{array}{lllllllllllllllllllll} & 3.902 & 3.918 & 3.934 & 3.916 & 3.912 & 3.828 & 3.892 & 3.850 & 3.894 & 3.890 & 3.904 & 3.892 & 3.896 & 3.890 & 3.894 & 3.876 & 3.906 & 3.876 & 3.900\end{array}$

$\mathrm{CaTh}(\mathrm{PO})_{2}$

$\mathrm{P}+\mathrm{Y}+\mathrm{REE}(\mathrm{apfu})$ $\begin{array}{lllllllllllllllllllllllll}1.058 & 1.048 & 1.053 & 1.059 & 1.062 & 1.049 & 1.038 & 1.027 & 1.045 & 1.051 & 1.091 & 1.056 & 1.071 & 1.057 & 1.053 & 1.044 & 1.053 & 1.050 & 1.050 & 1.053 & 1.056 & 1.045 & 1.057 & 1.052\end{array}$

- 94.41696 .00696 .28895 .23795 .06296 .50496 .61495 .54297 .38597 .71996 .68896 .60596 .02496 .01697 .05596 .21896 .57296 .12396 .05795 .94695 .45796 .75095 .13796 .867

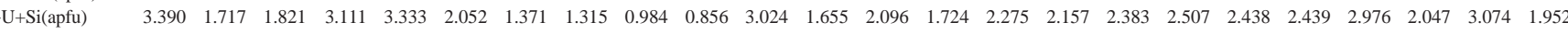

$(\mathrm{Ce}+\mathrm{La}+\mathrm{Nd}) / \mathrm{Th} \quad 33.16781 .00073 .09138 .143 \quad 34.26158 .214117 .000138 .833166 .800208 .50037 .14390 .66766 .91781 .50050 .68851 .93844 .94442 .78945 .16744 .77835 .34855 .06735 .39157 .786$ $\mathrm{Ce} / \mathrm{Th}$

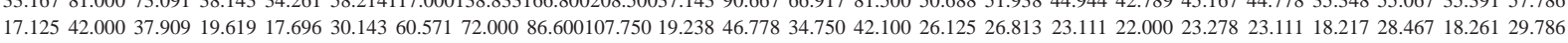




\begin{tabular}{|c|c|c|c|c|c|c|c|c|c|c|c|c|c|c|c|c|c|c|c|c|}
\hline \multirow[b]{2}{*}{ DataSet/Point } & \multicolumn{20}{|c|}{ Contact monazite Grain $(\mathrm{Cm})$ from chlorite schist } \\
\hline & $1 / 1$. & $2 / 1$. & $3 / 1$. & $4 / 1$. & $5 / 1$. & $6 / 1$. & $7 / 1$. & $8 / 1$. & $9 / 1$. & $10 / 1$. & $11 / 1$. & $12 / 1$. & $13 / 1$. & $14 / 1$. & $15 / 1$. & $16 / 1$. & $17 / 1$. & $18 / 1$. & $19 / 1$. & $20 / 1$. \\
\hline $\mathrm{Al}_{2} \mathrm{O}_{3}$ & 0.00 & 0.00 & 0.00 & 0.00 & 0.00 & 0.00 & 0.00 & 0.00 & 0.00 & 0.00 & 0.00 & 0.00 & 0.00 & 0.00 & 0.00 & 0.00 & 0.00 & 0.00 & 0.00 & 0.00 \\
\hline $\mathrm{SiO}_{2}$ & 0.21 & 0.16 & 0.21 & 0.19 & 0.18 & 0.43 & 0.19 & 0.16 & 0.14 & 0.25 & 0.22 & 0.15 & 0.15 & 0.15 & 0.16 & 0.34 & 0.15 & 0.15 & 0.16 & 0.16 \\
\hline $\mathrm{P}_{2} \mathrm{O}_{5}$ & 30.15 & 29.77 & 29.56 & 30.00 & 29.48 & 28.80 & 29.51 & 29.30 & 29.60 & 28.74 & 28.06 & 29.18 & 29.38 & 29.45 & 29.04 & 28.84 & 29.07 & 29.17 & 29.58 & 29.52 \\
\hline $\mathrm{CaO}$ & 0.15 & 0.13 & 0.15 & 0.15 & 0.16 & 0.24 & 0.15 & 0.08 & 0.07 & 0.11 & 0.15 & 0.09 & 0.11 & 0.11 & 0.10 & 0.12 & 0.07 & 0.08 & 0.20 & 0.06 \\
\hline $\mathrm{FeO}$ & 0.00 & 0.00 & 0.00 & 0.00 & 0.00 & 0.00 & 0.00 & 0.00 & 0.00 & 0.00 & 0.00 & 0.00 & 0.00 & 0.00 & 0.00 & 0.00 & 0.00 & 0.00 & 0.00 & 0.00 \\
\hline $\mathrm{Y}_{2} \mathrm{O}_{3}$ & 1.39 & 1.46 & 1.40 & 1.39 & 1.44 & 1.31 & 0.85 & 0.78 & 0.76 & 0.89 & 0.76 & 0.84 & 0.89 & 0.77 & 1.00 & 0.79 & 0.80 & 0.79 & 1.35 & 0.86 \\
\hline $\mathrm{La}_{2} \mathrm{O}_{3}$ & 15.72 & 15.15 & 15.48 & 15.83 & 15.07 & 15.26 & 16.30 & 16.37 & 16.88 & 16.39 & 16.38 & 16.10 & 16.46 & 17.16 & 16.00 & 16.23 & 16.24 & 16.53 & 15.49 & 16.12 \\
\hline $\mathrm{Ce}_{2} \mathrm{O}_{3}$ & 30.43 & 30.09 & 30.43 & 30.47 & 30.25 & 29.93 & 31.39 & 31.70 & 31.19 & 31.00 & 31.20 & 31.82 & 31.05 & 31.66 & 30.86 & 31.44 & 31.40 & 31.47 & 30.53 & 31.53 \\
\hline $\mathrm{Nd}_{2} \mathrm{O}_{3}$ & 13.48 & 13.83 & 13.60 & 13.66 & 13.66 & 13.24 & 13.44 & 13.20 & 12.92 & 13.01 & 13.11 & 13.32 & 13.13 & 12.97 & 13.44 & 13.25 & 13.18 & 13.17 & 13.43 & 13.36 \\
\hline $\mathrm{SmO}$ & 2.59 & 2.84 & 2.75 & 2.70 & 2.66 & 2.68 & 2.46 & 2.54 & 2.30 & 2.28 & 2.34 & 2.36 & 2.30 & 2.22 & 2.57 & 2.37 & 2.33 & 2.34 & 2.56 & 2.43 \\
\hline $\mathrm{Gd}_{2} \mathrm{O}_{3}$ & 1.65 & 1.70 & 1.59 & 1.55 & 1.68 & 1.61 & 1.32 & 1.30 & 1.26 & 1.35 & 1.24 & 1.31 & 1.27 & 1.28 & 1.43 & 1.19 & 1.26 & 1.29 & 1.48 & 1.36 \\
\hline $\mathrm{Dy}_{2} \mathrm{O}_{3}$ & 0.43 & 0.45 & 0.42 & 0.43 & 0.40 & 0.40 & 0.26 & 0.31 & 0.26 & 0.34 & 0.30 & 0.27 & 0.34 & 0.31 & 0.38 & 0.30 & 0.25 & 0.28 & 0.39 & 0.28 \\
\hline $\mathrm{Ho}_{2} \mathrm{O}_{3}$ & 0.00 & 0.00 & 0.00 & 0.00 & 0.00 & 0.00 & 0.00 & 0.00 & 0.00 & 0.00 & 0.00 & 0.00 & 0.00 & 0.00 & 0.00 & 0.00 & 0.00 & 0.00 & 0.00 & 0.00 \\
\hline $\mathrm{PbO}$ & 0.03 & 0.03 & 0.02 & 0.02 & 0.03 & 0.03 & 0.01 & 0.02 & 0.02 & 0.03 & 0.02 & 0.02 & 0.03 & 0.02 & 0.02 & 0.01 & 0.02 & 0.01 & 0.04 & 0.01 \\
\hline $\mathrm{ThO}_{2}$ & 0.10 & 0.10 & 0.07 & 0.00 & 0.18 & 0.12 & 0.09 & 0.11 & 0.06 & 0.07 & 0.14 & 0.09 & 0.09 & 0.05 & 0.10 & 0.08 & 0.05 & 0.05 & 0.15 & 0.09 \\
\hline $\mathrm{UO}_{2}$ & 0.27 & 0.31 & 0.26 & 0.28 & 0.27 & 0.27 & 0.17 & 0.19 & 0.19 & 0.27 & 0.18 & 0.17 & 0.31 & 0.20 & 0.25 & 0.19 & 0.19 & 0.18 & 0.26 & 0.19 \\
\hline Total & 100.04 & 99.53 & 99.32 & 100.12 & 98.90 & 97.78 & 99.57 & 99.47 & 99.08 & 98.07 & 97.44 & 99.29 & 98.90 & 99.70 & 98.82 & 98.60 & 98.37 & 98.82 & 99.09 & 99.34 \\
\hline iormula & (O) & $4(\mathrm{O})$ & $4(\mathrm{O})$ & $4(\mathrm{O})$ & $4(\mathrm{O})$ & $4(\mathrm{O})$ & $4(\mathrm{O})$ & $4(\mathrm{O})$ & $4(\mathrm{O})$ & $4(\mathrm{O})$ & $4(\mathrm{O})$ & $4(\mathrm{O})$ & $4(\mathrm{O})$ & $4(\mathrm{O})$ & $4(\mathrm{O})$ & $4(C$ & $4(\mathrm{O})$ & $4(\mathrm{O})$ & $4(\mathrm{O})$ & $4(\mathrm{O})$ \\
\hline $\mathrm{Ca}$ & 0.006 & 0.005 & 0.006 & 0.006 & 0.007 & 0.010 & 0.006 & 0.003 & 0.003 & 0.005 & 0.006 & 0.004 & 0.004 & 0.005 & 0.004 & 0.005 & 0.003 & 0.003 & 0.008 & 0.002 \\
\hline $\mathrm{Fe}$ & 0.000 & 0.000 & 0.000 & 0.000 & 0.000 & 0.000 & 0.000 & 0.000 & 0.000 & 0.000 & 0.000 & 0.000 & 0.000 & 0.000 & 0.000 & 0.000 & 0.000 & 0.000 & 0.000 & 0.000 \\
\hline $\mathrm{Sm}$ & 0.035 & 0.038 & 0.037 & 0.036 & 0.036 & 0.037 & 0.034 & 0.035 & 0.031 & 0.032 & 0.033 & 0.032 & 0.031 & 0.030 & 0.035 & 0.033 & 0.032 & 0.032 & 0.035 & 0.033 \\
\hline $\mathrm{Pb}$ & 0.000 & 0.000 & 0.000 & 0.000 & 0.000 & 0.000 & 0.000 & 0.000 & 0.000 & 0.000 & 0.000 & 0.000 & 0.000 & 0.000 & 0.000 & 0.000 & 0.000 & 0.000 & 0.000 & 0.000 \\
\hline $\mathrm{Al}$ & 0.000 & 0.000 & 0.000 & 0.000 & 0.000 & 0.000 & 0.000 & 0.000 & 0.000 & 0.000 & 0.000 & 0.000 & 0.000 & 0.000 & 0.000 & 0.0 & 0.000 & 0.000 & 0.000 & 0.000 \\
\hline Y & 0.029 & 0.031 & 0.029 & 0.029 & 0.030 & 0.028 & 0.018 & 0.017 & 0.016 & 0.019 & 0.017 & 0.018 & 0.019 & 0.016 & 0.021 & 0.017 & 0.017 & 0.017 & 0.028 & 0.018 \\
\hline $\mathrm{La}$ & 0.226 & 0.220 & 0.226 & 0.228 & 0.220 & 0.226 & 0.238 & 0.240 & 0.247 & 0.244 & 0.247 & 0.237 & 0.242 & 0.251 & 0.236 & 0.240 & 0.240 & 0.243 & 0.226 & 0.235 \\
\hline $\mathrm{Ce}$ & 0.434 & 0.434 & 0.440 & 0.436 & 0.439 & 0.440 & 0.454 & 0.461 & 0.453 & 0.458 & 0.467 & 0.464 & 0.452 & 0.459 & 0.452 & 0.461 & 0.461 & 0.460 & 0.442 & 0.457 \\
\hline $\operatorname{Pr}$ & 0.049 & 0.050 & 0.049 & 0.049 & 0.050 & 0.051 & 0.049 & 0.049 & 0.049 & 0.049 & 0.050 & 0.052 & 0.049 & 0.048 & 0.051 & 0.050 & 0.049 & 0.048 & 0.050 & 0.049 \\
\hline $\mathrm{Nd}$ & 0.188 & 0.194 & 0.192 & 0.191 & 0.194 & 0.190 & 0.190 & 0.187 & 0.183 & 0.187 & 0.192 & 0.190 & 0.187 & 0.183 & 0.192 & 0.190 & 0.189 & 0.188 & 0.190 & 0.189 \\
\hline Gd & 0.021 & 0.022 & 0.021 & 0.020 & 0.022 & 0.022 & 0.017 & 0.017 & 0.017 & 0.018 & 0.017 & 0.017 & 0.017 & 0.017 & 0.019 & 0.016 & 0.017 & 0.017 & 0.019 & 0.018 \\
\hline Dy & 0.005 & 0.006 & 0.005 & 0.005 & 0.005 & 0.005 & 0.003 & 0.004 & 0.003 & 0.004 & 0.004 & 0.003 & 0.004 & 0.004 & 0.005 & 0.0 & 0.003 & 0.004 & 0.005 & 0.004 \\
\hline Ho & 0.0 & 0.00 & & 0.00 & 0.0 & & 0.0 & 0.000 & 0.0 & 0.000 & 0.000 & 0.000 & 0.000 & 0.000 & 0.000 & 0.000 & 0.000 & 0.000 & 0.000 & 0.000 \\
\hline $\mathrm{Si}$ & 0.008 & 0.006 & 0.008 & 0.007 & 0.007 & 0.017 & 0.007 & 0.006 & 0.006 & 0.010 & 0.009 & 0.006 & 0.006 & 0.006 & 0.006 & 0.014 & 0.006 & 0.006 & 0.006 & 0.006 \\
\hline Th & 0.001 & 0.001 & 0.001 & 0.000 & 0.002 & 0.001 & 0.001 & 0.001 & 0.001 & 0.001 & 0.001 & 0.001 & 0.001 & 0.000 & 0.001 & 0.001 & 0.000 & 0.000 & 0.001 & 0.001 \\
\hline $\mathrm{U}$ & 0.002 & 0.003 & 0.002 & 0.002 & 0.002 & 0.002 & 0.002 & 0.002 & 0.002 & 0.002 & 0.002 & 0.002 & 0.003 & 0.002 & 0.002 & 0.002 & 0.002 & 0.002 & 0.002 & 0.002 \\
\hline $\mathrm{P}$ & 0.996 & 0.992 & 0.989 & 0.993 & 0.990 & 0.980 & 0.988 & 0.985 & 0.993 & 0.981 & 0.972 & 0.984 & 0.990 & 0.987 & 0.984 & 0.979 & 0.987 & 0.987 & 0.991 & 0.990 \\
\hline Total & 2.001 & 2.004 & 2.006 & 2.004 & 2.005 & 2.010 & 2.007 & 2.008 & 2.003 & 2.010 & 2.017 & 2.009 & 2.005 & 2.008 & 2.009 & 2.011 & 2.007 & 2.007 & 2.006 & 2.005 \\
\hline $\mathrm{D}$ & 0.780 & 0.793 & 0.792 & 0.784 & 0.797 & 0.800 & 0.782 & 0.782 & 0.762 & 0.782 & 0.797 & 0.788 & 0.775 & 0.771 & 0.789 & 0.785 & 0.778 & 0.776 & 0.793 & 0.777 \\
\hline
\end{tabular}

XBrb

Xhut

XLREE

XHREE

$\mathrm{XYPO}_{4}$

$\mathrm{Th} / \mathrm{U}$

Th (ppb)

$\mathrm{U}$ (ppb)

$\mathrm{Ce} / \mathrm{Sm}$

$\mathrm{Ca}+\mathrm{Si} /(\mathrm{Th}+\mathrm{U}+\mathrm{Pb})$

$\mathrm{REE}+\mathrm{P}+\mathrm{Y}$

$\mathrm{Th}+\mathrm{U}+\mathrm{SI}$

$\mathrm{Y}(\mathrm{ppm})$

$\mathrm{Si}+\mathrm{P}$

U+th+LREE+

HREE

$\mathrm{Ca}+\mathrm{Si}$

$\mathrm{U}+\mathrm{Th}+\mathrm{Pb}$

$\mathrm{La}^{*}$

Y+HREE

$\mathrm{Ca}+\mathrm{Th}+\mathrm{U}$

$2 \mathrm{ThSiO}_{4}$

$2 \mathrm{REEPO}_{4}$

$\mathrm{CaTh}(\mathrm{PO})_{2}$

$\mathrm{P}+\mathrm{Y}+\mathrm{REE}$ (apfu) $\mathrm{Th}+\mathrm{U}+\mathrm{Si}$ (apfu)

$(\mathrm{Ce}+\mathrm{La}+$

$\begin{array}{llllllllllllllllllll}0.016 & 0.014 & 0.016 & 0.016 & 0.017 & 0.026 & 0.016 & 0.009 & 0.008 & 0.012 & 0.016 & 0.010 & 0.012 & 0.012 & 0.011 & 0.013 & 0.008 & 0.008 & 0.021 & 0.006\end{array}$ $\begin{array}{llllllllllllllllllll}-0.003 & -0.002 & -0.004 & -0.005 & -0.003 & -0.008 & -0.005 & -0.001 & -0.001 & -0.002 & -0.004 & -0.002 & -0.001 & -0.003 & -0.001 & -0.003 & -0.001 & -0.002 & -0.006 & 0.000\end{array}$

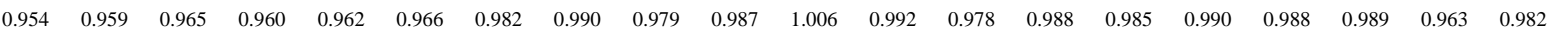
$\begin{array}{llllllllllllllllllll}0.005 & 0.006 & 0.005 & 0.005 & 0.005 & 0.005 & 0.003 & 0.004 & 0.003 & 0.004 & 0.004 & 0.003 & 0.004 & 0.004 & 0.005 & 0.004 & 0.003 & 0.004 & 0.005 & 0.004\end{array}$ \begin{tabular}{llllllllllllllllllll}
1.024 & 1.023 & 1.018 & 1.022 & 1.020 & 1.008 & 1.006 & 1.002 & 1.009 & 1.000 & 0.989 & 1.002 & 1.009 & 1.003 & 1.005 & 0.995 & 1.004 & 1.003 & 1.019 & 1.008 \\
\hline
\end{tabular}

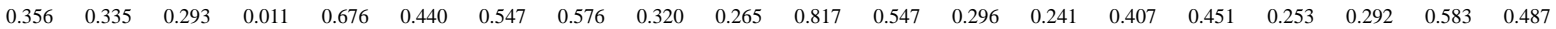
$\begin{array}{llllllllllllllllllll}0.846 & 0.898 & 0.649 & 0.026 & 1.610 & 1.041 & 0.827 & 0.952 & 0.534 & 0.631 & 1.273 & 0.818 & 0.801 & 0.427 & 0.881 & 0.729 & 0.418 & 0.445 & 1.335 & 0.818\end{array}$ $\begin{array}{llllllllllllllllllll}2.402 & 2.710 & 2.244 & 2.464 & 2.411 & 2.393 & 1.531 & 1.672 & 1.689 & 2.411 & 1.575 & 1.513 & 2.737 & 1.795 & 2.191 & 1.636 & 1.672 & 1.540 & 2.314 & 1.698\end{array}$

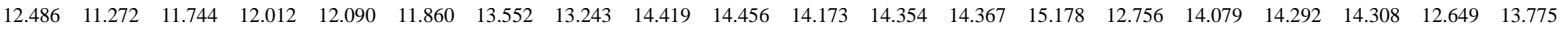

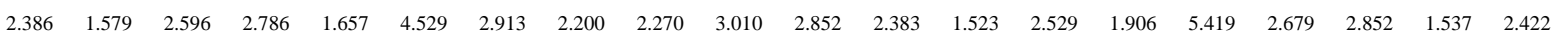

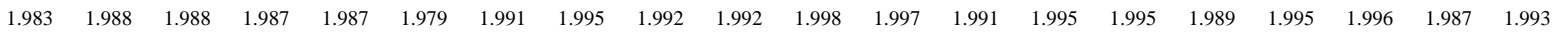

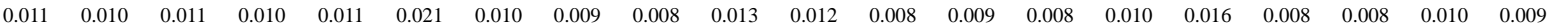

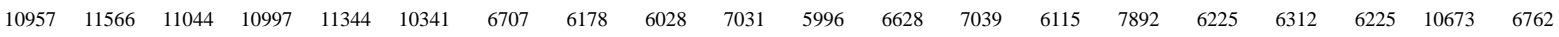

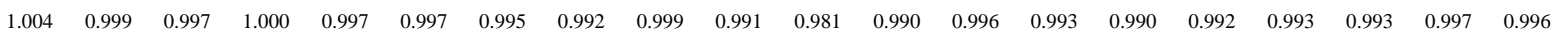

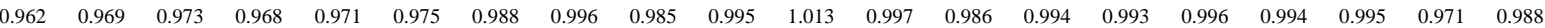

$\begin{array}{llllllllllllllllllll}0.014 & 0.012 & 0.015 & 0.014 & 0.014 & 0.027 & 0.013 & 0.010 & 0.008 & 0.015 & 0.016 & 0.010 & 0.010 & 0.011 & 0.011 & 0.019 & 0.009 & 0.009 & 0.015 & 0.009\end{array}$ $\begin{array}{llllllllllllllllllllll}0.003 & 0.004 & 0.003 & 0.003 & 0.004 & 0.004 & 0.003 & 0.003 & 0.002 & 0.003 & 0.003 & 0.003 & 0.004 & 0.002 & 0.003 & 0.003 & 0.002 & 0.002 & 0.004 & 0.003\end{array}$

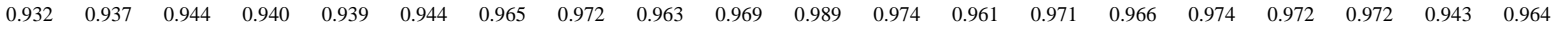
$\begin{array}{llllllllllllllllllll}0.034 & 0.036 & 0.035 & 0.034 & 0.035 & 0.033 & 0.021 & 0.021 & 0.019 & 0.023 & 0.020 & 0.021 & 0.023 & 0.020 & 0.026 & 0.021 & 0.020 & 0.020 & 0.033 & 0.022 \\ 0 & 0.00 & 0.009 & 0.009 & 0.01 & 0.014 & 0.009 & 0.06 & 0.05 & 0.008 & 0.00 & 0.006 & 0.008 & 0.07 & 0.007 & 0.08 & 0.005 & 0.005 & 0.02 & 0.005\end{array}$ $\begin{array}{lllllllllllllllllllll}0.009 & 0.009 & 0.009 & 0.009 & 0.011 & 0.014 & 0.009 & 0.006 & 0.005 & 0.008 & 0.009 & 0.006 & 0.008 & 0.007 & 0.007 & 0.008 & 0.005 & 0.005 & 0.012 & 0.005\end{array}$

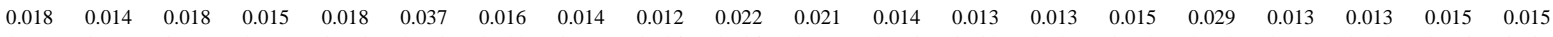

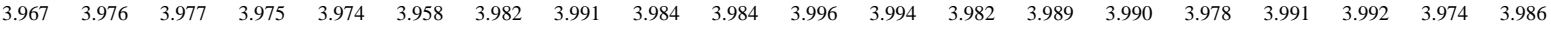

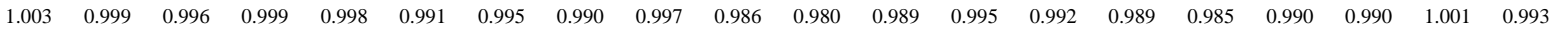

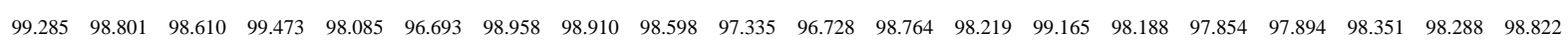

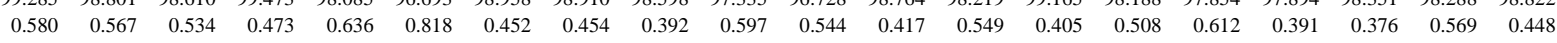




\begin{tabular}{|c|c|c|c|c|c|c|c|c|c|c|c|c|c|c|c|c|c|c|c|c|}
\hline \multirow[b]{2}{*}{ DataSet/Point } & \multicolumn{20}{|c|}{ Contact monazite Grain $(\mathrm{Cm})$ from chlorite schist } \\
\hline & $21 / 1$. & $22 / 1$. & $23 / 1$. & $24 / 1$. & $25 / 1$. & $26 / 1$. & $27 / 1$. & $28 / 1$ & $29 / 1$. & $30 / 1$. & $31 / 1$. & $32 / 1$. & $33 / 1$. & $34 / 1$. & $35 / 1$. & $36 / 1$. & $37 / 1$. & $38 / 1$. & $39 / 1$. & $40 / 1$ \\
\hline $\mathrm{Al}_{2} \mathrm{O}_{3}$ & 0.00 & 0.00 & 0.00 & 0.00 & 0.00 & 0.00 & 0.00 & 0.00 & 0.00 & 0.00 & 0.00 & 0.00 & 0.00 & 0.00 & 0.00 & 0.00 & 0.00 & 0.00 & 0.00 & 0.00 \\
\hline $\mathrm{SiO}_{2}$ & 0.17 & 0.16 & 0.15 & 0.16 & 0.18 & 0.24 & 0.16 & 0.17 & 0.16 & 0.18 & 0.17 & 0.17 & 0.20 & 0.18 & 0.16 & 0.16 & 0.18 & 0.18 & 0.16 & 0.24 \\
\hline $\mathrm{P}_{2} \mathrm{O}_{5}$ & 29.71 & 29.72 & 30.22 & 30.11 & 30.40 & 30.64 & 30.64 & 30.80 & 30.46 & 30.58 & 30.79 & 31.05 & 30.46 & 30.66 & 30.80 & 30.51 & 30.06 & 30.60 & 30.65 & 30.86 \\
\hline $\mathrm{CaO}$ & 0.09 & 0.10 & 0.09 & 0.08 & 0.07 & 0.11 & 0.11 & 0.09 & 0.08 & 0.10 & 0.10 & 0.10 & 0.33 & 0.26 & 0.07 & 0.38 & 0.25 & 0.12 & 0.14 & 0.21 \\
\hline $\mathrm{FeO}$ & 0.00 & 0.00 & 0.00 & 0.00 & 0.00 & 0.00 & 0.00 & 0.00 & 0.00 & 0.00 & 0.00 & 0.00 & 0.00 & 0.00 & 0.00 & 0.00 & 0.00 & 0.00 & 0.00 & 0.00 \\
\hline $\mathrm{Y}_{2} \mathrm{O}_{3}$ & 0.90 & 0.86 & 0.80 & 0.80 & 0.83 & 0.90 & 0.74 & 0.63 & 0.80 & 0.86 & 0.65 & 1.02 & 0.91 & 0.91 & 0.73 & 0.88 & 0.89 & 0.86 & 1.18 & 0.86 \\
\hline $\mathrm{La}_{2} \mathrm{O}_{3}$ & 16.37 & 16.19 & 16.78 & 16.21 & 16.61 & 16.72 & 16.30 & 16.98 & 16.63 & 16.40 & 16.46 & 15.62 & 15.96 & 15.51 & 16.36 & 16.28 & 16.06 & 16.30 & 15.44 & 16.42 \\
\hline $\mathrm{Ce}_{2}^{2} \mathrm{O}_{3}$ & 31.09 & 31.29 & 31.21 & 31.59 & 31.48 & 30.95 & 30.75 & 31.79 & 31.01 & 30.83 & 31.47 & 30.65 & 29.90 & 29.91 & 30.76 & 30.30 & 30.31 & 30.46 & 29.99 & 30.82 \\
\hline $\mathrm{Nd}_{2}^{2} \mathrm{O}_{3}^{3}$ & 13.31 & 13.39 & 12.94 & 13.54 & 13.07 & 13.12 & 13.47 & 12.89 & 13.04 & 12.96 & 13.09 & 13.75 & 13.08 & 13.22 & 13.30 & 12.53 & 12.84 & 12.93 & 13.30 & 12.84 \\
\hline $\mathrm{SmO}$ & 2.54 & 2.41 & 2.37 & 2.52 & 2.44 & 2.48 & 2.49 & 2.20 & 2.41 & 2.32 & 2.29 & 2.66 & 2.42 & 2.50 & 2.49 & 2.30 & 2.33 & 2.34 & 2.62 & 2.42 \\
\hline $\mathrm{Gd}_{2} \mathrm{O}_{3}$ & 1.40 & 1.34 & 1.31 & 1.37 & 1.27 & 1.49 & 1.42 & 1.18 & 1.42 & 1.34 & 1.17 & 1.48 & 1.27 & 1.34 & 1.34 & 1.29 & 1.27 & 1.46 & 1.51 & 1.39 \\
\hline $\mathrm{Dy}_{2} \mathrm{O}_{3}$ & 0.32 & 0.34 & 0.32 & 0.29 & 0.31 & 0.32 & 0.27 & 0.24 & 0.28 & 0.29 & 0.26 & 0.35 & 0.31 & 0.23 & 0.28 & 0.34 & 0.31 & 0.32 & 0.36 & 0.29 \\
\hline $\mathrm{Ho}_{2} \mathrm{O}_{3}$ & 0.00 & 0.00 & 0.00 & 0.00 & 0.00 & 0.00 & 0.00 & 0.00 & 0.00 & 0.00 & 0.00 & 0.00 & 0.00 & 0.00 & 0.00 & 0.00 & 0.00 & 0.00 & 0.00 & 0.00 \\
\hline $\mathrm{PbO}$ & 0.02 & 0.02 & 0.02 & 0.02 & 0.02 & 0.03 & 0.02 & 0.02 & 0.01 & 0.02 & 0.01 & 0.03 & 0.05 & 0.06 & 0.02 & 0.05 & 0.02 & 0.04 & 0.02 & 0.01 \\
\hline $\mathrm{ThO}_{2}$ & 0.05 & 0.14 & 0.10 & 0.09 & 0.05 & 0.09 & 0.16 & 0.09 & 0.06 & 0.08 & 0.24 & 0.03 & 0.99 & 1.27 & 0.21 & 0.99 & 0.15 & 0.17 & 0.21 & 0.11 \\
\hline $\mathrm{UO}_{2}{ }_{2}^{2}$ & 0.26 & 0.18 & 0.23 & 0.17 & 0.20 & 0.32 & 0.24 & 0.13 & 0.20 & 0.25 & 0.11 & 0.26 & 0.21 & 0.25 & 0.20 & 0.23 & 0.18 & 0.43 & 0.20 & 0.17 \\
\hline Total & 99.58 & 99.64 & 99.99 & 100.33 & 100.49 & 100.93 & 100.15 & 100.75 & 99.91 & 99.69 & 100.20 & 100.68 & 99.41 & 99.67 & 100.11 & 99.65 & 98.22 & 99.66 & 99.28 & 100.02 \\
\hline ormula & $4(\mathrm{O})$ & $4(\mathrm{O})$ & $4(\mathrm{O})$ & $4(\mathrm{O})$ & $4(\mathrm{O})$ & $4(\mathrm{O})$ & $4(\mathrm{O})$ & $4(\mathrm{O})$ & $4(\mathrm{O})$ & $4(\mathrm{O})$ & $4(\mathrm{O})$ & $4(\mathrm{O})$ & $4(C$ & $4(\mathrm{O})$ & $4(\mathrm{O})$ & $4(\mathrm{O})$ & $4(C$ & $4(\mathrm{O})$ & $4(\mathrm{O})$ & 4( \\
\hline $\mathrm{Ca}$ & 0.004 & 0.004 & 0.004 & 0.003 & 0.003 & 0.004 & 0.005 & 0.004 & 0.004 & 0.004 & 0.004 & 0.004 & 0.014 & 0.011 & 0.003 & 0.016 & 0.010 & 0.005 & 0.006 & 0.009 \\
\hline $\mathrm{Fe}$ & 0.000 & 0.000 & 0.000 & 0.000 & 0.000 & 0.000 & 0.000 & 0.000 & 0.000 & 0.000 & 0.000 & 0.000 & 0.000 & 0.000 & 0.000 & 0.000 & 0.000 & 0.000 & 0.000 & 0.000 \\
\hline $\mathrm{Sm}$ & 0.035 & 0.033 & 0.032 & 0.034 & 0.033 & 0.033 & 0.033 & 0.029 & 0.032 & 0.031 & 0.031 & 0.035 & 0.032 & 0.034 & 0.033 & 0.031 & 0.032 & 0.031 & 0.035 & 0.032 \\
\hline $\mathrm{Pb}$ & 0.000 & 0.000 & 0.000 & 0.000 & 0.000 & 0.000 & 0.000 & 0.000 & 0.000 & 0.000 & 0.000 & 0.000 & 0.000 & 0.001 & 0.000 & 0.000 & 0.000 & 0.000 & 0.000 & 0.000 \\
\hline $\mathrm{Al}$ & 0.000 & 0.000 & 0.000 & 0.000 & 0.000 & 0.000 & 0.000 & 0.000 & 0.000 & 0.000 & 0.000 & 0.000 & 0.000 & 0.000 & 0.000 & 0.000 & 0.000 & 0.000 & 0.000 & 0.000 \\
\hline Y & 0.019 & 0.018 & 0.017 & 0.017 & 0.017 & 0.019 & 0.015 & 0.013 & 0.017 & 0.018 & 0.013 & 0.021 & 0.019 & 0.019 & 0.015 & 0.018 & 0.019 & 0.018 & 0.024 & 0.018 \\
\hline $\mathrm{La}$ & 0.238 & 0.235 & 0.242 & 0.233 & 0.238 & 0.238 & 0.233 & 0.241 & 0.239 & 0.235 & 0.235 & 0.221 & 0.229 & 0.222 & 0.233 & 0.234 & 0.234 & 0.234 & 0.222 & 0.234 \\
\hline $\mathrm{Ce}$ & 0.449 & 0.452 & 0.446 & 0.452 & 0.448 & 0.437 & 0.436 & 0.449 & 0.442 & 0.439 & 0.445 & 0.431 & 0.427 & 0.425 & 0.436 & 0.432 & 0.438 & 0.434 & 0.427 & 0.435 \\
\hline $\mathrm{Pr}$ & 0.048 & 0.050 & 0.049 & 0.048 & 0.050 & 0.049 & 0.048 & 0.050 & 0.047 & 0.050 & 0.048 & 0.049 & 0.047 & 0.048 & 0.048 & 0.048 & 0.048 & 0.049 & 0.050 & 0.047 \\
\hline $\mathrm{Nd}$ & 0.187 & 0.188 & 0.180 & 0.189 & 0.181 & 0.181 & 0.186 & 0.177 & 0.181 & 0.180 & 0.181 & 0.189 & 0.182 & 0.183 & 0.184 & 0.174 & 0.181 & 0.179 & 0.185 & 0.177 \\
\hline $\mathrm{Gd}$ & 0.018 & 0.018 & 0.017 & 0.018 & 0.016 & 0.019 & 0.018 & 0.015 & 0.018 & 0.017 & 0.015 & 0.019 & 0.016 & 0.017 & 0.017 & 0.017 & 0.017 & 0.019 & 0.019 & 0.018 \\
\hline Dy & 0.004 & 0.004 & 0.004 & 0.004 & 0.004 & 0.004 & 0.003 & 0.003 & 0.004 & 0.0 & 0.003 & 0.004 & 0.00 & 0.003 & 0.003 & 0.004 & 0.004 & 0.004 & 0.005 & 0.004 \\
\hline Ho & 0.000 & 0.000 & 0.000 & 0.000 & 0.000 & 0.000 & 0.000 & 0.000 & 0.000 & 0.000 & 0.000 & 0.000 & 0.000 & 0.000 & 0.000 & 0.000 & 0.000 & 0.000 & 0.000 & 0.000 \\
\hline $\mathrm{Si}$ & 0.007 & 0.006 & 0.006 & 0.006 & 0.007 & 0.009 & 0.006 & 0.006 & 0.006 & 0.007 & 0.007 & 0.007 & 0.008 & 0.007 & 0.006 & 0.006 & 0.007 & 0.007 & 0.006 & 0.009 \\
\hline Th & 0.000 & 0.001 & 0.001 & 0.001 & 0.000 & 0.001 & 0.001 & 0.001 & 0.000 & 0.001 & 0.002 & 0.000 & 0.009 & 0.011 & 0.002 & 0.009 & 0.001 & 0.001 & 0.002 & 0.001 \\
\hline $\mathrm{U}$ & 0.002 & 0.002 & 0.002 & 0.001 & 0.002 & 0.003 & 0.002 & 0.001 & 0.002 & 0.002 & 0.001 & 0.002 & 0.002 & 0.002 & 0.002 & 0.002 & 0.002 & 0.004 & 0.002 & 0.001 \\
\hline P & 0.992 & 0.992 & 0.999 & 0.995 & 0.999 & 1.000 & 1.006 & 1.005 & 1.004 & 1.007 & 1.008 & 1.009 & 1.005 & 1.008 & 1.009 & 1.005 & 1.005 & 1.007 & 1.010 & 1.008 \\
\hline Total & 2.004 & 2.004 & 1.999 & 2.001 & 1.999 & 1.997 & 1.994 & 1.995 & 1.996 & 1.994 & 1.993 & 1.992 & 1.995 & 1.991 & 1.992 & 1.996 & 1.997 & 1.993 & 1.992 & 1.993 \\
\hline D & 0.773 & 0.776 & 0.758 & 0.771 & 0.759 & 0.757 & 0.756 & 0.747 & 0.753 & 0.751 & 0.749 & 0.761 & 0.769 & 0.767 & 0.748 & 0.769 & 0.764 & 0.754 & 0.762 & 0.752 \\
\hline
\end{tabular}

XBrb

Xhut

XLREE

XHREE

$\mathrm{XYPO}_{4}$

$\mathrm{Th} / \mathrm{U}$

Th (ppb)

$\mathrm{U}$ (ppb)

$\mathrm{Ce} / \mathrm{Sm}$

$\mathrm{Ca}+\mathrm{Si} /(\mathrm{Th}+\mathrm{U}+\mathrm{Pb})$

$\mathrm{REE}+\mathrm{P}+\mathrm{Y}$

$\mathrm{Th}+\mathrm{U}+\mathrm{SI}$

$\mathrm{Y}(\mathrm{ppm})$

$\mathrm{Si}+\mathrm{P}$

U+th+LREE+

HREE

$\mathrm{Ca}+\mathrm{Si}$

$\mathrm{U}+\mathrm{Th}+\mathrm{Pb}$

La*

Y+HREE

$\mathrm{Ca}+\mathrm{Th}+\mathrm{U}$

$2 \mathrm{ThSiO}_{4}$

$2 \mathrm{REEPO}_{4}$

$\mathrm{CaTh}(\mathrm{PO})_{2}$

$\mathrm{P}+\mathrm{Y}+\mathrm{REE}$ (apfu) $\mathrm{Th}+\mathrm{U}+\mathrm{Si}$ (apfu)

$(\mathrm{Ce}+\mathrm{La}+\mathrm{Nd}) / \mathrm{Th}$

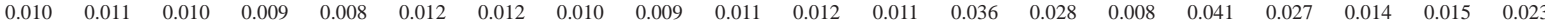
$\begin{array}{rrrrrrrrrrrrrrrrrrrrr}-0.001 & -0.002 & -0.001 & -0.001 & -0.001 & -0.001 & -0.001 & -0.002 & -0.001 & -0.001 & -0.001 & -0.002 & -0.004 & 0.004 & 0.001 & -0.006 & -0.009 & 0.001 & -0.003 & -0.008\end{array}$

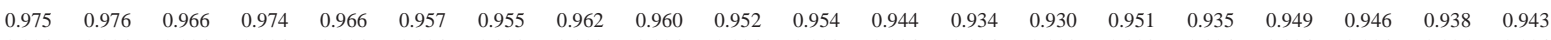
$\begin{array}{lllllllllllllllllllll}0.004 & 0.004 & 0.004 & 0.004 & 0.004 & 0.004 & 0.003 & 0.003 & 0.004 & 0.004 & 0.003 & 0.004 & 0.004 & 0.003 & 0.003 & 0.004 & 0.004 & 0.004 & 0.005 & 0.004\end{array}$

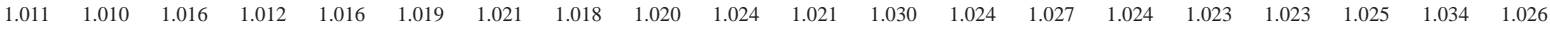

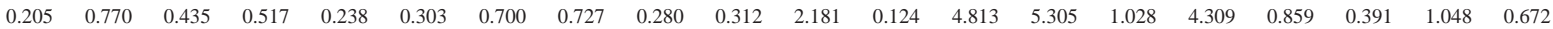

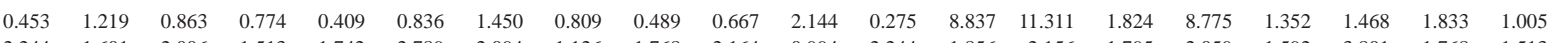
$\begin{array}{llllllllllllllllllll}2.244 & 1.601 & 2.006 & 1.513 & 1.742 & 2.789 & 2.094 & 1.126 & 1.768 & 2.164 & 0.994 & 2.244 & 1.856 & 2.156 & 1.795 & 2.059 & 1.592 & 3.801 & 1.768 & 1.513\end{array}$

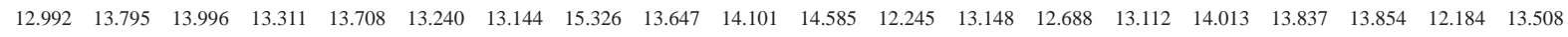

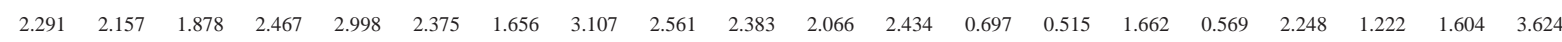

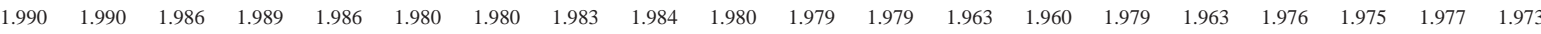

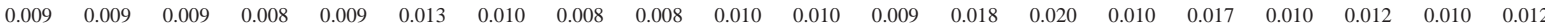

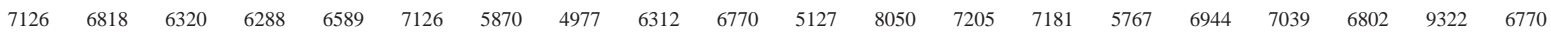

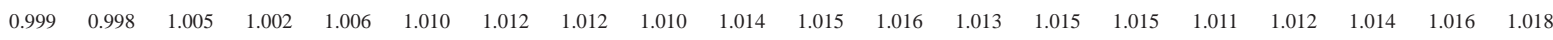

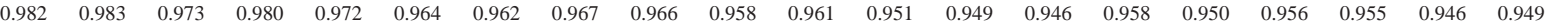

\begin{tabular}{llllllllllllllllllll}
0.010 & 0.011 & 0.010 & 0.010 & 0.010 & 0.014 & 0.011 & 0.010 & 0.010 & 0.011 & 0.011 & 0.011 & 0.022 & 0.018 & 0.009 & 0.022 & 0.018 & 0.012 & 0.012 & 0.018 \\
\hline
\end{tabular} $\begin{array}{lllllllllllllllllllll}0.003 & 0.003 & 0.003 & 0.002 & 0.002 & 0.004 & 0.004 & 0.002 & 0.002 & 0.003 & 0.003 & 0.003 & 0.011 & 0.014 & 0.004 & 0.011 & 0.003 & 0.006 & 0.004 & 0.003\end{array}$

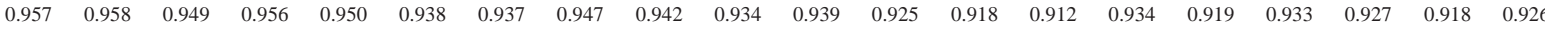
$\begin{array}{llllllllllllllllllll}0.023 & 0.022 & 0.021 & 0.020 & 0.021 & 0.023 & 0.019 & 0.016 & 0.020 & 0.021 & 0.017 & 0.025 & 0.023 & 0.022 & 0.019 & 0.022 & 0.023 & 0.022 & 0.029 & 0.021\end{array}$ $\begin{array}{llllllllllllllllllll}0.007 & 0.007 & 0.007 & 0.006 & 0.005 & 0.008 & 0.008 & 0.006 & 0.006 & 0.007 & 0.007 & 0.007 & 0.025 & 0.024 & 0.007 & 0.027 & 0.013 & 0.010 & 0.009 & 0.011\end{array}$

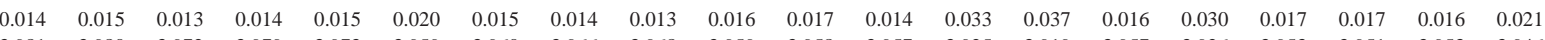

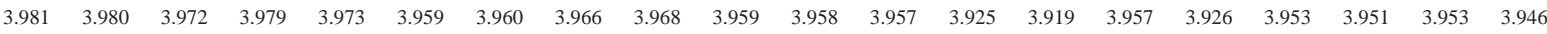

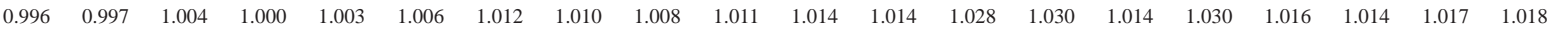

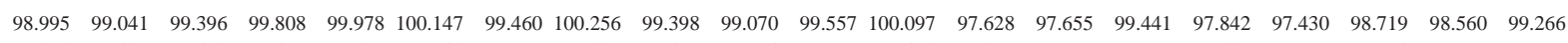

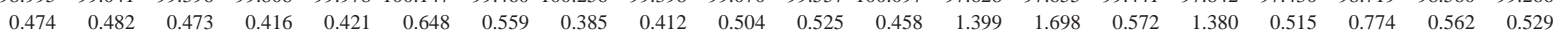




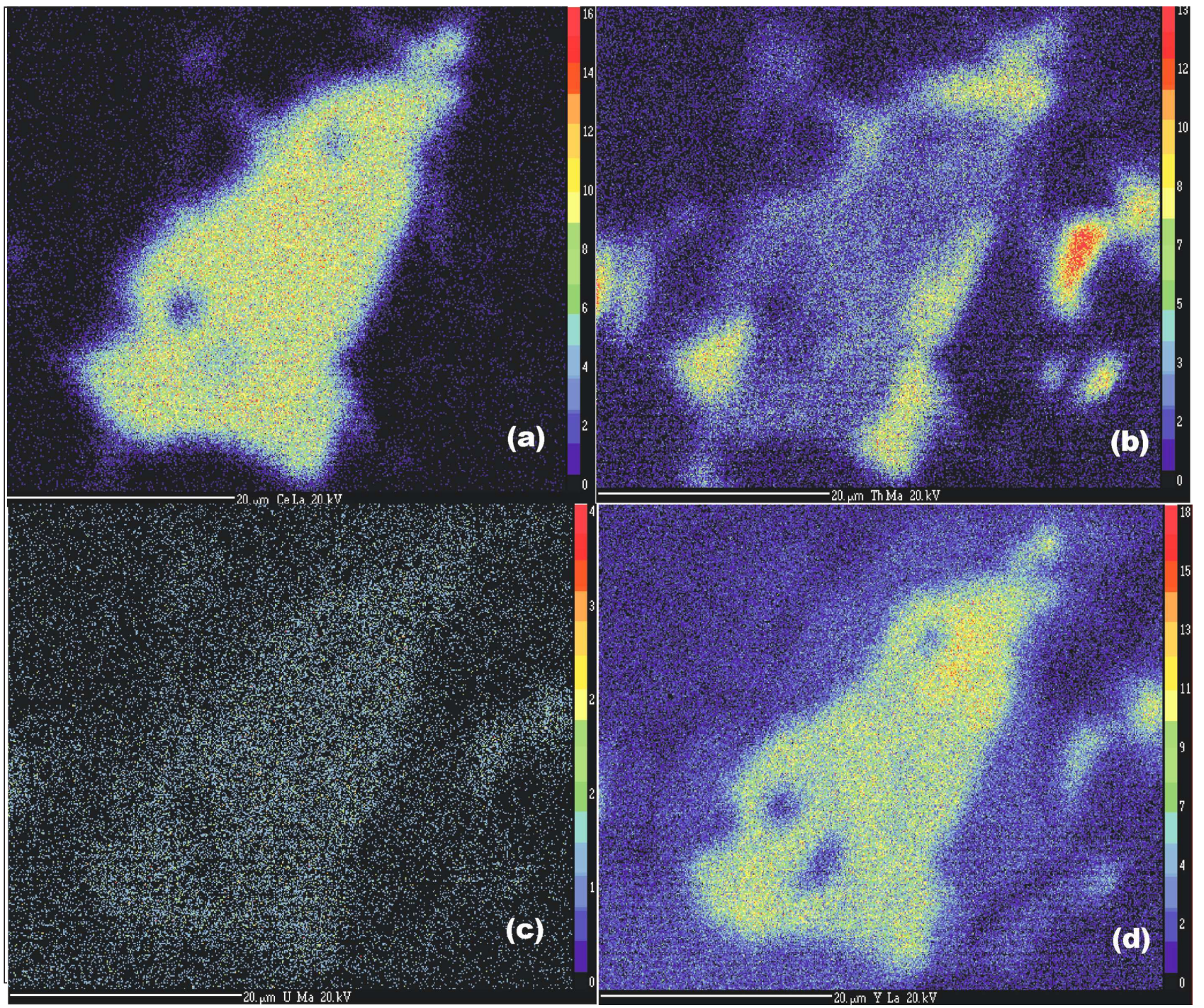

Fig.8. X-ray elemental maps of $C e(a), T h(b), U(c)$, and $Y(d)$ of grain5. Note resorbed nature of the grain especially in the Th and $Y$ maps which indicate that originally this grain was larger with the high Th and higher Y fragments on right central part of the X-ray maps being part of the same grain.

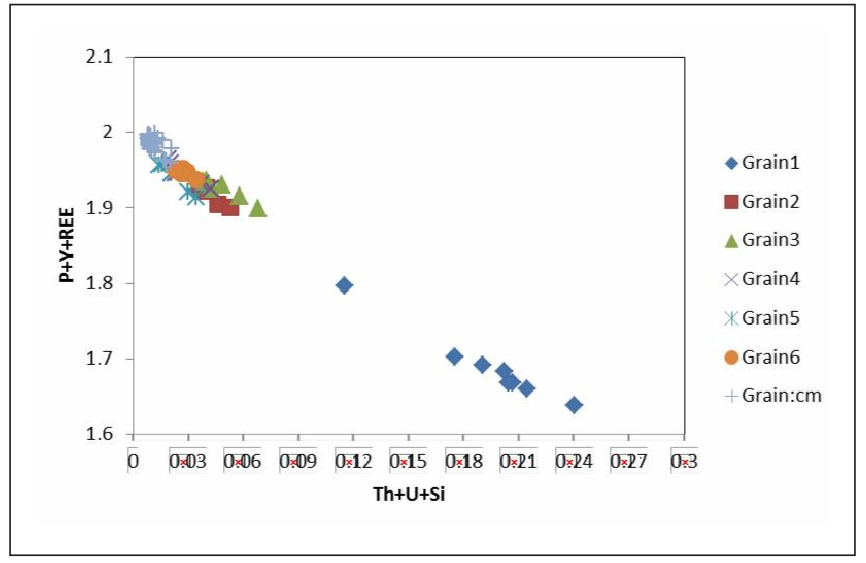

Fig.9. A plot of $T h+U+S i$ versus $P+Y+R E E$. Note: Segregation of contact monazite at one end. Inclusion monazite is depleted in REE as compared to matrix grains while it has higher $T h+U$ than matrix grains.

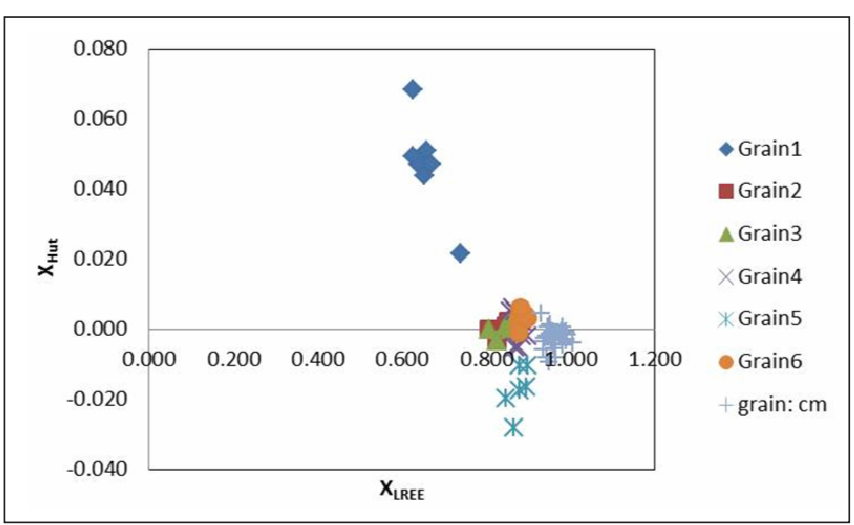

Fig.10. A plot of $X_{L R E E}$ versus $X_{H U T}$ Note: Contact monazite grains value occurs on the extreme end of LREE showing enrichment. Grain1 is depleted in LREE as compared to matrix grains and show higher huttonite content. 


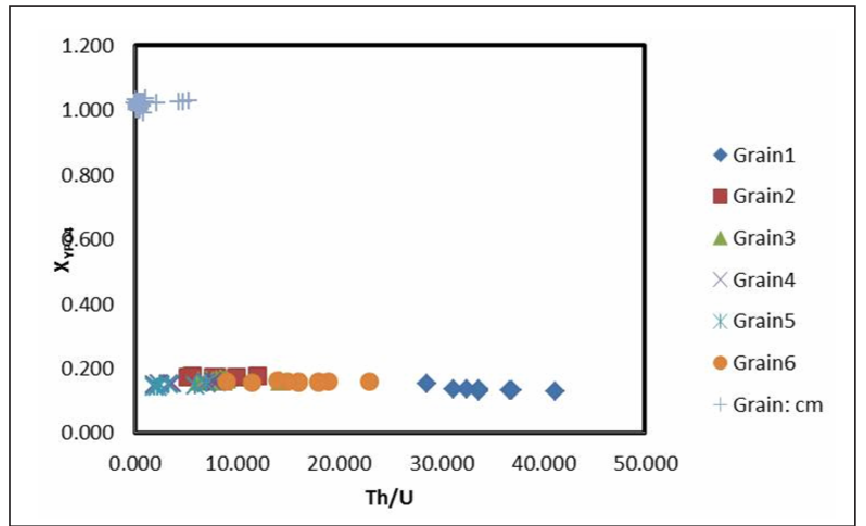

Fig.11. A plot of $X_{Y P O 4}$ versus $T h / U$. Note: All six grains have almost similar $Y$ content but $T h / U$ ratio is significantly higher in grain1 and contact monazite grain has highest $Y$ content and lowest $T h / U$.
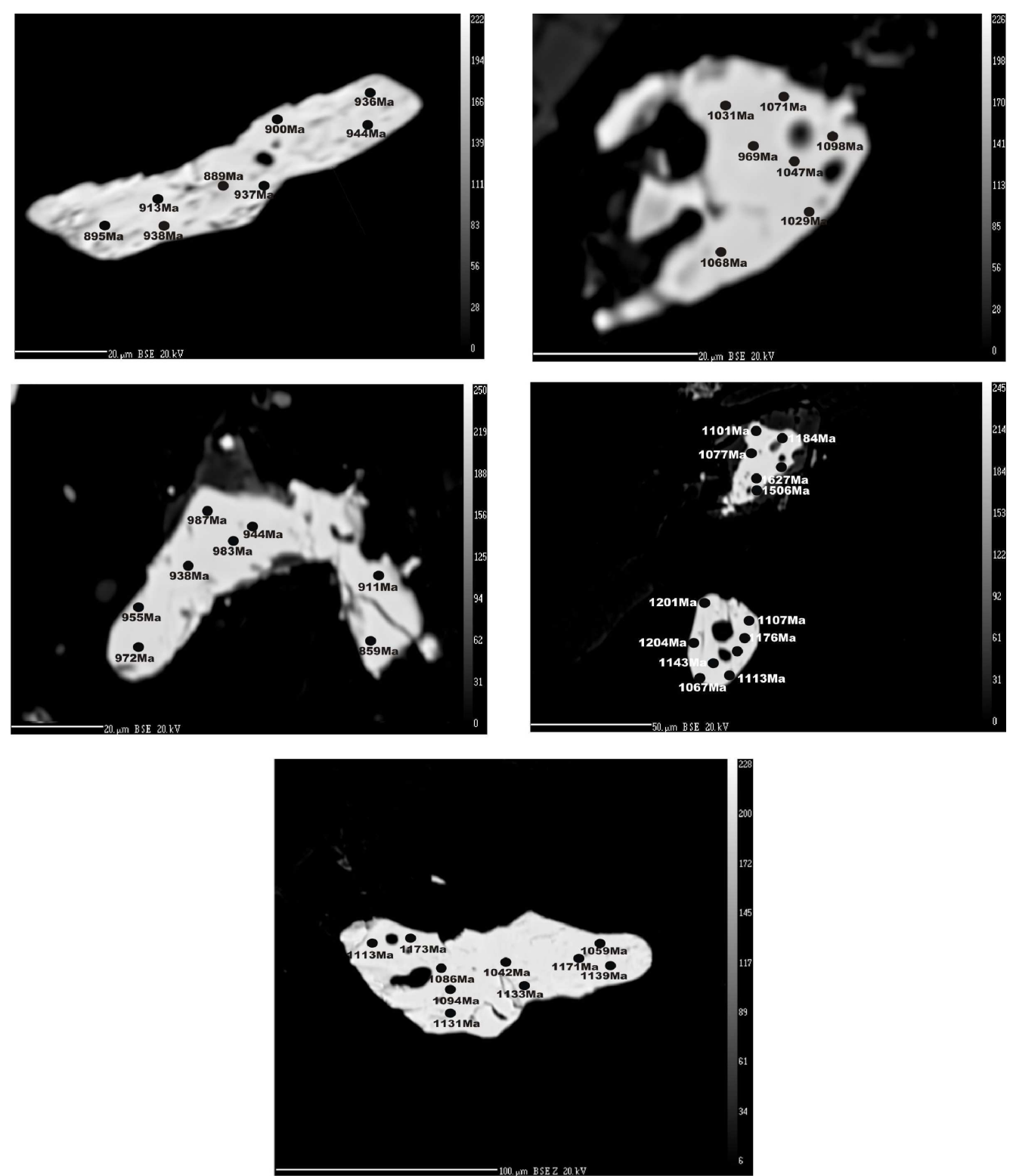

Fig.13. BSE image of six monazite grains annoted with the spot ages.

Fig.12. A plot of Variation between brabantite and huttonite. Note: grain 1 has positive brabantite and huttonite component while other grains only have huttonite component. It has lower brabantite content comparative to grain1 but it is higher than matrix grains.
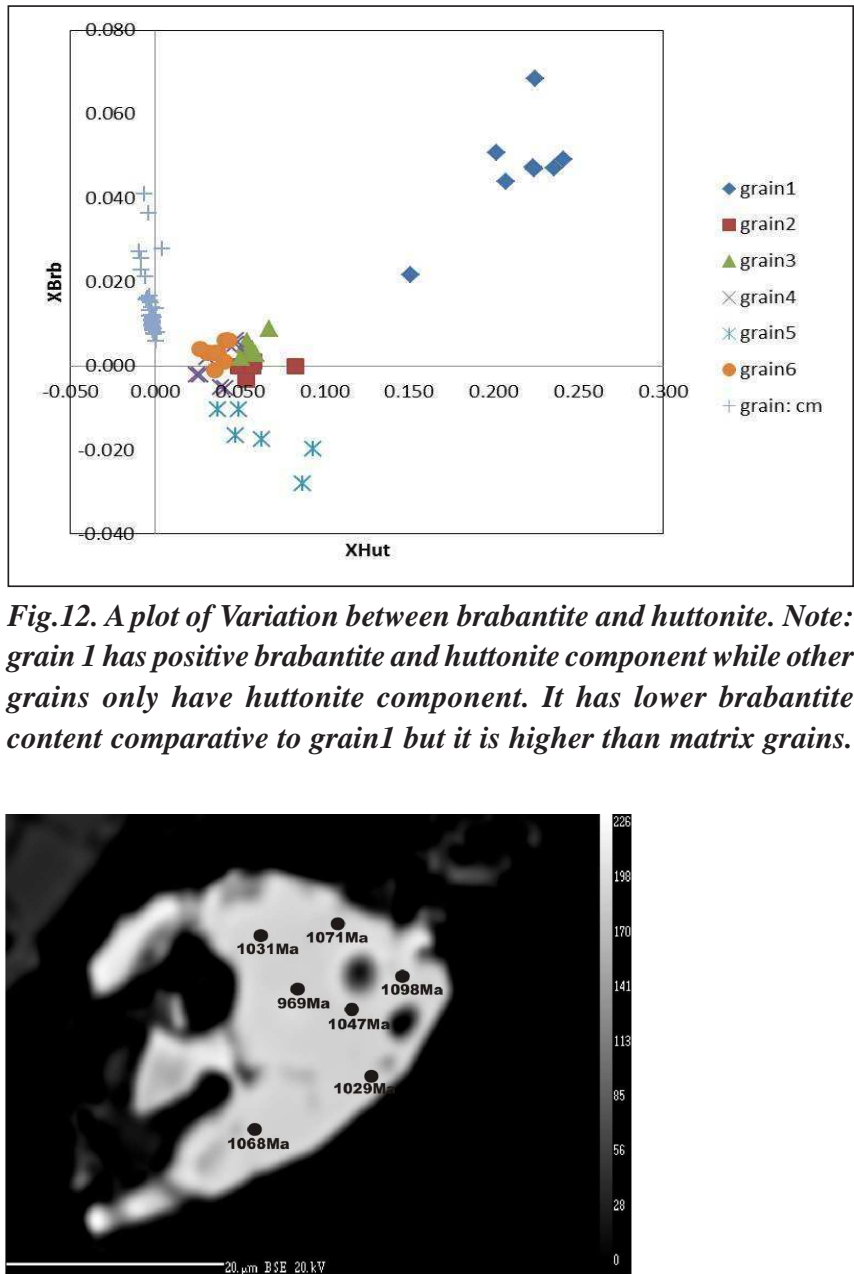


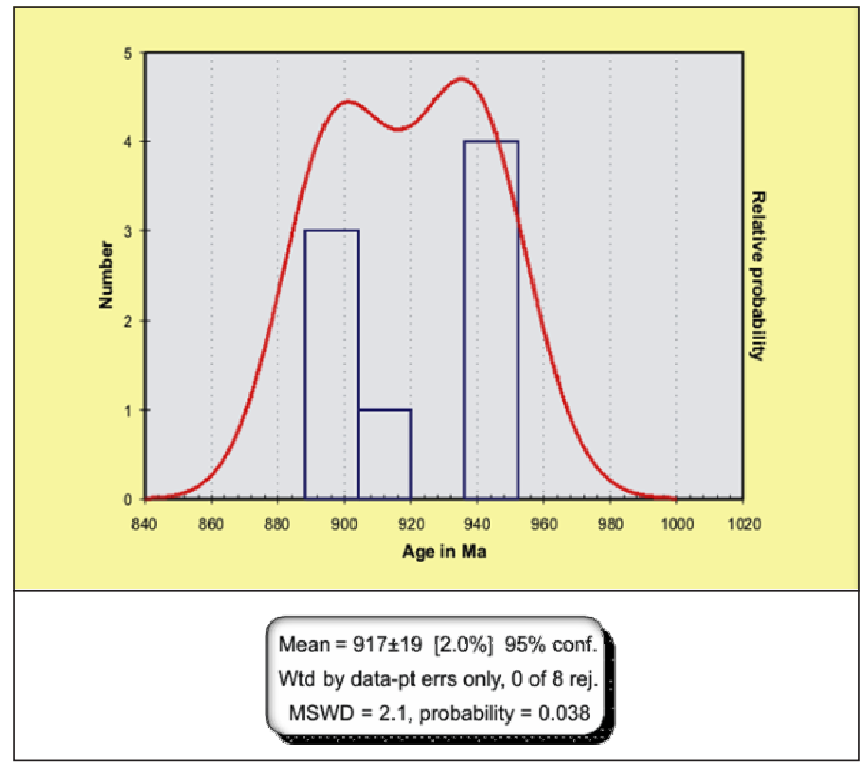

Fig14. Probability density plot of grain 1 gives an age of $917 \pm 19$ Ma.

(Fig. 14). The point age data of grains 2, 3, 4, 5 and 6 gives two distinct age populations that are $941 \pm 23 \mathrm{Ma}$ and $1100 \pm 20 \mathrm{Ma}$ (Fig. 15). The older age constitutes $75 \%$ of the ages while the younger age is within the range of age estimated from the inclusion grain. The ages of 1506 and $1627 \mathrm{Ma}$ are from a grain which is anhedral and altering and the low $\mathrm{Th}$ in respect of these points possibly reflects open system for this element.

\section{Contact zone monazite (Sample $\mathrm{KH}-2$ )}

Monazites in this sample are comparatively of larger size. The analyzed grain is anhedral with irregular grain boundary and has length $\sim 200 \mu \mathrm{m}$. Monazite is unzoned and distinctly different from all monazites of garnet-staurolite schist. This grain is enriched in LREE while it is depleted in huttonite content (Fig.10). It has strikingly higher $\mathrm{X}_{\mathrm{YPO} 4}$ than other monazites while it has $\mathrm{Th} / \mathrm{U}$ content close to 0 (Fig.11). It has lower brabantite content comparative to

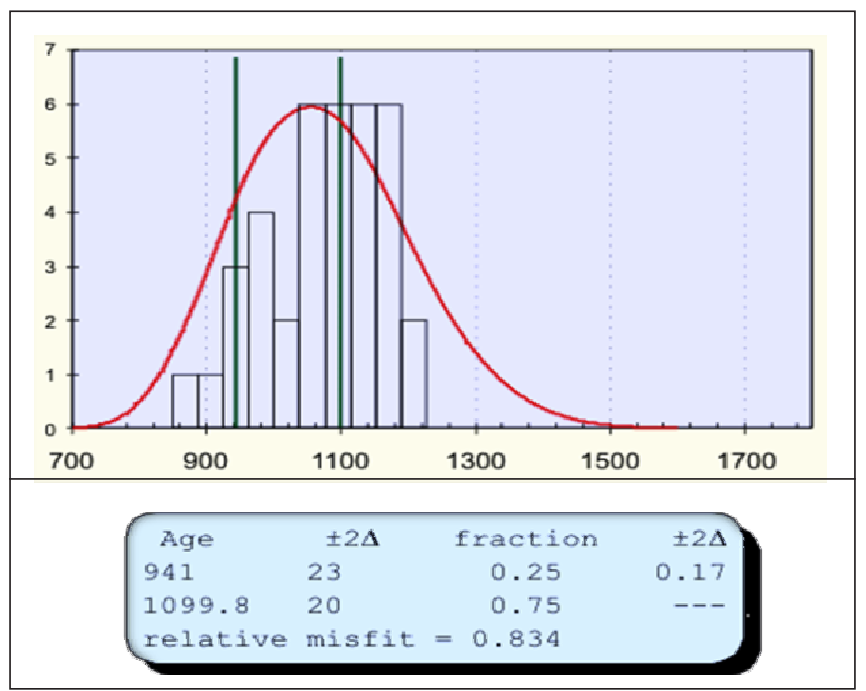

Fig.15. Probability density plot of grains 2, 3, 4, 5 and 6 gives two distinct age populations that are, $941 \pm 23 \mathrm{Ma}$ and $1100 \pm 20 \mathrm{Ma}$.

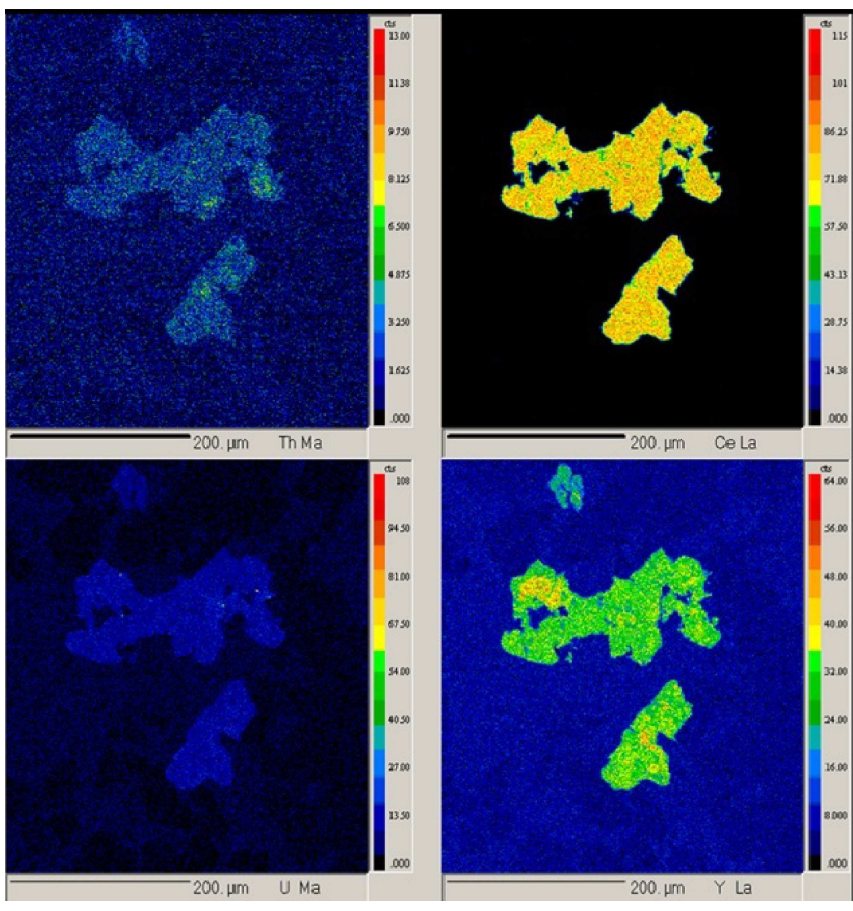

Fig.16. Elemental map of Th, Ce, $U$ and $Y$ of contact monazite grain.

grain1 but it is higher than matrix grains (Fig. 12). It also shows highest value of $\mathrm{P}+\mathrm{Y}+\mathrm{REE}$ which signifies its enrichment in REE (Fig. 9). Fig.16 shows the elemental mapping of Th, $\mathrm{Ce}, \mathrm{U}$ and $\mathrm{Y}$ which shows grain is also a Ce-monazite but has higher Ce content comparative to other monazites. The most significant character about this monazite is that it is significantly depleted in Th content (Fig. 16).

A 40 point's average on $2 \sigma$ level gives the statistically analyzed age around $649 \pm 40 \mathrm{Ma}$ (Fig.17).

\section{Discussion}

Several modes of monazite formation have been proposed which

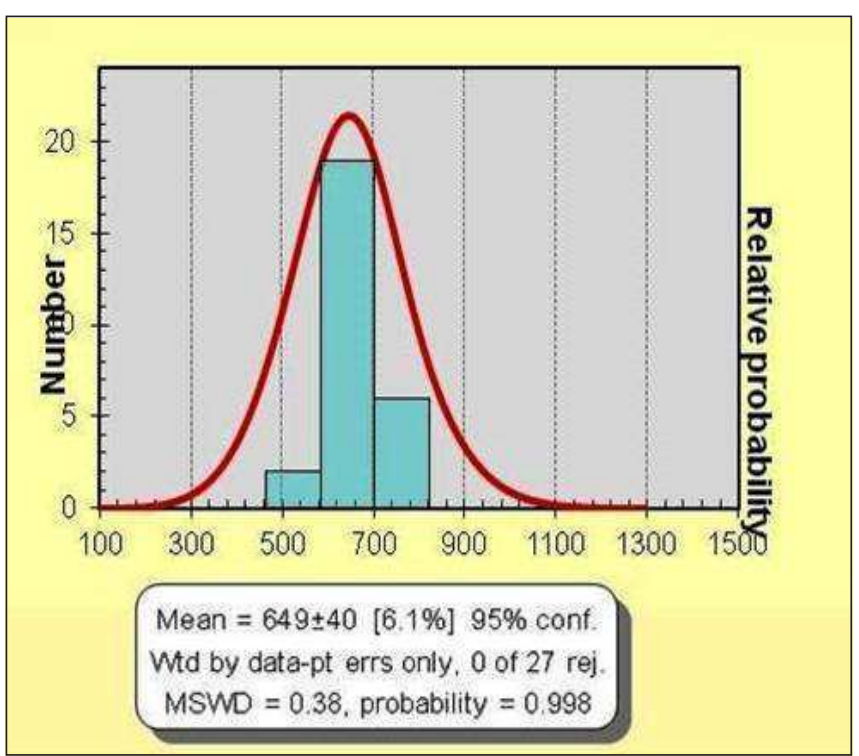

Fig.17. A 40 point's average on $2 \sigma$ level gives the statistically analyzed age around $649 \pm 40 \mathrm{Ma}$. 


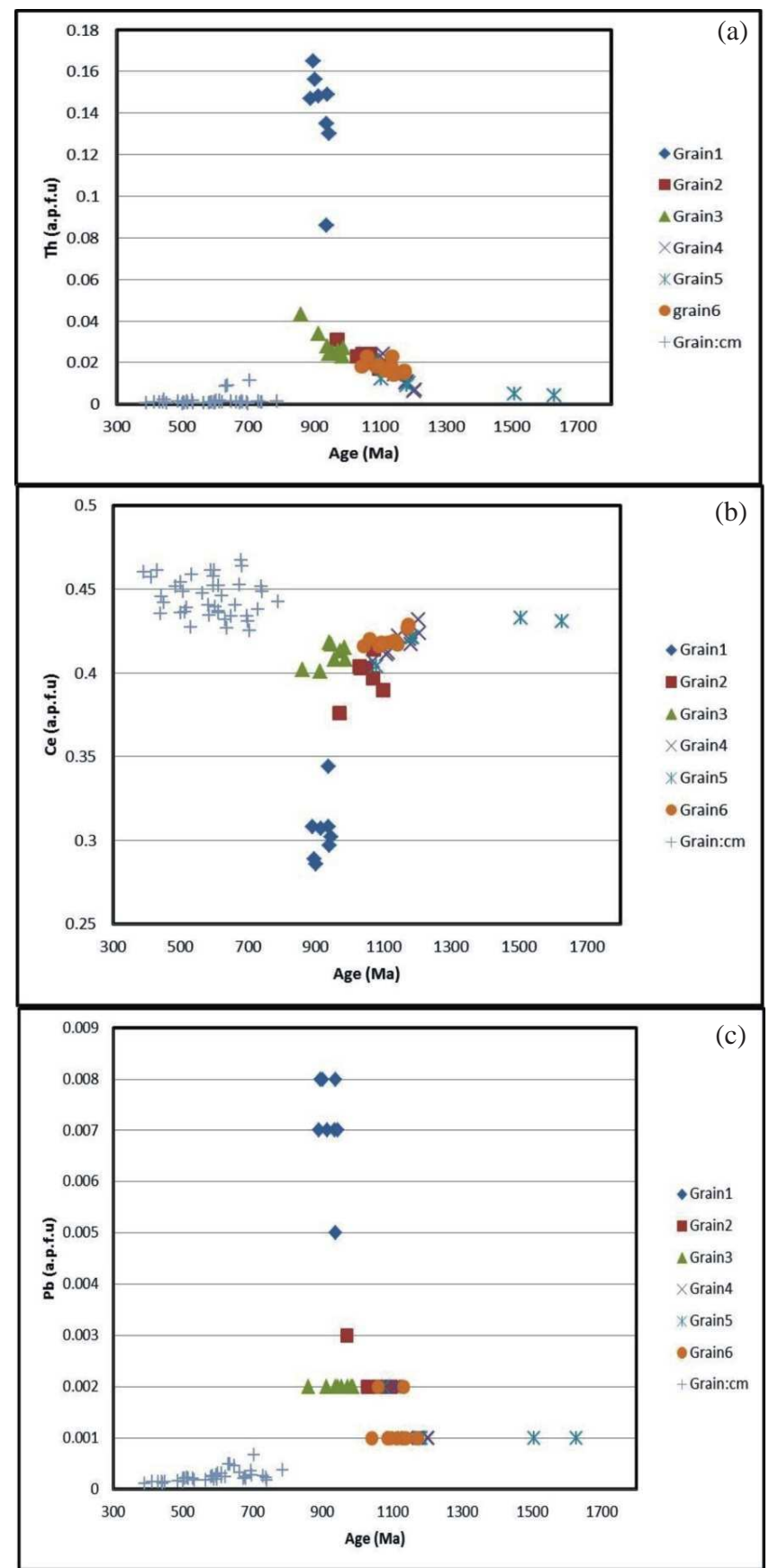

Fig.18. A plot of Thorium (a), Cerium (b), and Lead (c) variation versus age of seven monazite grains.

include heterogeneous reaction (which include the solid state growth or recrystallization under changed pressure-temperature conditions or metamorphic growth), precipitation from fluids, dissolution and/ or reprecipitation under fluid influx (hydrothermal mode), annealing etc. and in hydrothermal influenced growth it often partially or totally resets the U-Th-Pb systematic (Spear and Pyle, 2002; Ayers et al. 2006). Precipitation of REE and monazite growth in contact aureoles has been attributed to fluid mobilization (Rasmussen et al. 2001; Rasmussen and Fletcher, 2002). As in this study monazite has been occasionally used to date the hydrothermal event (Ayers et al., 2006; Rasmussen et al., 2007). Since there is strong control of fluids in monazite growth during contact metamorphism, chemical distinctions between monazite formed dominantly by solid state transformation and that in a contact aureole can be expected. Ayers et al. (2006) noted lower $\mathrm{Si}$, $\mathrm{Th}, \mathrm{Pb}$ and $\mathrm{U}$ and higher REE in monazite proximal to the contact zone in the Birch Creek Pluton aureole in eastern California which they attributed to the dissolution and reprecipitation of monazite by the infiltrating fluids which was corroborated by the oxygen isotope compositions of the proximal and distal monazites. Thus, besides temperature fluids appear to strongly influence the monazite chemistry in contact metamorphic setting. It has been noticed that the brabantite component increases away from a contact metamorphism aureole (Ayers et al. 2006). Th (or U) $+\mathrm{Ca}=2 \mathrm{REE}$ represent the brabantite substitution. A plot of brabantite versus huttonite shows that monazite from contact zone of $\mathrm{KCB}$ and regional metamorphic monazite from Golwa- Gangutana area are chemically distinct (Fig. 12). The huttonite content is lowest in contact monazite. The nature of zoning in two types of monazite is also different. Grain 4 and grain 5 of matrix show patchy and partial zoning which are possibly due to partial resetting of the U-Th-Pb systems (Townsend et al., 2000; Ayers et al. 2006, 2013) or due to recrystalization during retrograde metamorphism. The composite monazite from the lowest grade portion of the contact aureole present in south central Maine (Wing et al., 2003) also show similar trend as of contact monazite of KCB. The composite monazites are zoned monazite with inclusion free core and inclusion rich rim which is a result of recrystallization to varying degrees (Wing et al., 2003).

All monazites of both samples are Ce-rich (Table 1). However significant and contrasting chemical variations are clearly distinct and three sets of monazite compositions can be identified. Since, three age domains are indicated the $\mathrm{Ce}, \mathrm{Th}$ and $\mathrm{Pb}$ values are plotted against age (Fig. 18) to assess the chemical variations. Inclusion grain1 has highest $\mathrm{Ce}$ and matrix grains 2,3,4,5 and 6 in the garnet staurolite schist contain lower Th (Fig. 18a). The two outlier values of 1506 and $1627 \mathrm{Ma}$ are not considered on account of very low Th and it is inferred that these either represent a detrital component or disequilibrium composition. These two ages are from rim area of grain 5 which is anhedral and appears to be resorbed. The monazite from the contact mineralized zone (Khetri) plots distinctly with lowest Th. Behavior of $\mathrm{Ce}$ is inverse of the Th concentration with the contact monazite having highest $\mathrm{Ce}$ (Fig. 18b). Pb plot mirrors the Th behavior (Fig. 18c). In view of this, $\mathrm{Ce} / \mathrm{Th}$ can be considered as a function to segregate the three composition domains. The $\mathrm{Ce} / \mathrm{Th}$ values of grain 1 range from 1.75-4.00 while that of the matrix grains of the garnet staurolite schist vary from 10-70 (excluding the outliers) with most of the values between 20-40 while the contact monazite shows much higher ratios (>240) of these two elements (Table 1).

The inclusion monazite (grain 1) and the younger age of the matrix monazites provide a well constrained age of 920-940 Ma which is close to the age of the peak regional metamorphism in the area (Pant et al., 2008). The metamorphic conditions for the Barrovian metamorphism have been estimated to be $550^{\circ} \pm 50^{\circ} \mathrm{C}$ and $550^{\circ}-650^{\circ} \mathrm{C}$ with pressure range of 300 to $500 \mathrm{MPa}$ during M1 and M2 phases, respectively (e.g. Sarkar and Dasgupta, 1980; Lal and Ackermand, 1981; Sharma, 1988; Kundu et al., 2004; Pant et al. 2008). Formation of cordierite and biotite as a contact metamorphic effect in Khetri and its association with the $\mathrm{Cu}$-sulfide deposit suggests mineralization to be hydrothermally controlled. Chemical dating of monazite indicates age of $\sim 650 \mathrm{Ma}$ for this mineralization. Association of chemically distinct monazite in this well constrained contact metamorphic assemblage shows that the monazite formed in such 
setting can be distinguished using $\mathrm{Ce} / \mathrm{Th}$ ratios in comparison to the monazites of the regional metamorphic assemblages.

\section{Acknowledgements}

Amitava Kundu and Sonalika Joshi are acknowledged for electron microprobe analysis. R. Bhardwaj helped in sampling form Khetri copper mine. This work forms part of a Department of Science and Technology (DST) funded project entitled 'Correlation of magmatic and metamorphic evolution of the North and South Delhi fold Belts: Constraints from chemical age dating using zircon/allanite/thorite/ monazite".

\section{References}

Ayers, J.C., Crombie, S., Loflin, M., Miller, C.F. and Luo, Y., 2013, Country rock monazite response to intrusion of the search light pluton, Southern Nevada. American Journal of Science, v. 313, pp. 345-394, DOI 10.2475/ 04.2013 .04

Ayers, J.C., Loflin, M., Miller, C.F., Barton, M.D., and Coath, C.D., 2006, In situ oxygen isotope analysis of monazite as a monitor of fluid infiltration during contact metamorphism: Birch Creek Pluton aureole, White Mountains, eastern California. Geology, v. 34, no. 8, pp. 653-656, http:/ /dx.doi.org/10.1130/G22185.1

Banerjee, A.K., 1976, Tectonics and ore location in northeastern Rajasthan, India. Indian Mineralogist, v. 29, pp. 1-24

Basu, A.K., 1986, Geology of parts of the Bundelkhand massif, central India. Geological Survey of India, Records, v. 117(2), pp. 61-124.

Chakrabarti, B. and Gupta, G.P., 1992, Stratigraphy and structure of the North Delhi Basin. Geological Survey of India, Records, v. 124, pp. 5-9.

Copeland, P., Parrish, R.R. and Harrison, T.M., 1988, Identi?cation of inherited radiogenic $\mathrm{Pb}$ in monazite and its implications for $\mathrm{U}-\mathrm{Pb}$ systematics. Nature, v. 333, pp. 760-763.

Das Gupta, S.P., 1968, The structural history of the Khetri Copper Belt, Jhunjhunu and Sikar districts, Rajasthan. Memoir Geological Survey of India, v. 98, 170 p.

Das Gupta, S.P., 1964, Structural evolution of Khetri Copper Belt, Rajasthan, India, Report $22^{\text {nd }}$ International Congress, New Delhi, v. 4, pp. 357-373.

Gupta, P., Guha, D.B. and Chattopadhyay, B., 1998, Basement-cover relationship in the Khetri Copper Belt and the emplacement mechanism of the granite massifs, Rajasthan. Journal Geological Society of India, v. 52, pp. 417-432.

Heron, A.M., 1923, Geology of western Jaipur. Record Geological Survey of India, v. 54, pp. 345-397.

Janots, E., Engi, M., Rubatto, D., Berger, A., Gregory, C. and Rahn, M., 2009, Metamorphic rates in collisional orogeny from in situ allanite and monazite dating. Geology, v. 37, pp. 11-14, doi: 10.1130/G25192A.1

Janots, E., Engi, M., Berger, A., Allaz, J., Schwarz, J.O. and Spandler, C., 2008, Prograde metamorphic sequence of REE minerals in pelitic rocks of the Central Alps: Implications for allanite-monazite-xenotime phase relations from 250 to $610^{\circ} \mathrm{C}$. Journal of Metamorphic Geology, v. 26, pp. 509-526, doi: 10.1111/j.1525-1314.2008.00774.x.

Knight, J., Joy, S., Lowe, J., Cameron, J., Merrillees, J., Nag, S., Shah, N., Dua, G., Jhala, K., 2002, The Khetri copper belt, Rajasthan: Iron oxide copper-gold terrane in the Proterozoic of NW India. In. Prter TM (ed) Hydrothermal Iron Oxide Copper-Gold \& related deposits, a global perspective, vol. 2, PGC Publishing, Adelaide, pp. 321-341.

Kolitsch, U. and Holtstam, D., 2004, Crystal chemistry of REEXO4 compounds ( $\mathrm{X}=\mathrm{P}$, as, V). II. Review of REEXO4 compounds and their stability fields. European Journal of Mineralogy, v. 16, pp. 117-126.

Krenn, E. and Finger, F., 2007, Formation of monazite and rhabdophane at the expense of allanite during Alpine low temperature retrogression of metapelitic basement rocks from Crete, Greece: Microprobe data and geochronological implications. Lithos, v. 95, pp. 130-147

Kundu, A., Kazim, K. and Sharma, S., 2004, Metamorphism in north Delhi Fold Belt- a case study from southwest Haryana and adjacent parts of Rajasthan. Indian Minerals, v. 58, pp.17-26.

Lal, R.K. and Ackermand, D., 1981, Phase petrology and polyphase andalusitesillimanite type regional metamorphism in pelitic schist of the area around Akwali, Khetri Copper Belt, Rajasthan, India. N Jb Mineral Abh, v. 141, pp. 161-185.

Lal, R.K. and Shukla, R.S., 1975, Low-pressure regional metamorphism in the northern portion of the Khetri Copper Belt, Rajasthan, India. N Jb Mineral Abh, v. 124, pp. 294-325.

Ludwig, K.R., 2003, User's manual for Isoplot/Ex, v3.0, a geochronological toolkit for Microsoft Excel. Berkeley Geochronological Centre Special Publication, no. $4,70 \mathrm{p}$.

Montel, J.M., Foret, S., Veschambre, M., Nicollet, C. and Provost, A., 1996, Electron microprobe dating in monazite. Chemical Geology, v. 131, pp.3753.

Pant, N.C., Amitava Kundu and Sonalika Joshi, 2008, Age of Metamorphism of Delhi Supergroup rocks- electron microprobe age from Mahendragarh district, Haryana. Journal Geological Society of India, v. 72, pp. 365372.

Pant, N.C., Kundu, A., Joshi, S., Dey, A., Bhandari, A. and Joshi, A., 2009, Chemical dating of monazite: testing of an analytical protocol against independently dated standards, Indian Journal of Geosciences, v. 63, pp. 311-318.

Pouchour, J.L. and Pichoir, F., 1991 Quantitative analysis of homogeneous or stratified micro volumes applying the model "PAP". In: Heinrich KFJ and Newbury DE (eds) Electron probe quantification. Plenum Press, New York, pp. 31-75.

Prasad, S., Verma, K.P., Singh, R., Powar, M.M., Dasgupta, G., Kachroo, K. and Sharma, S., 1994, Report on stratigraphic correlation and regional chemical appraisal of Delhi Supergroup in southern parts of Haryana. Unpublished Report Geological Survey of India.

Rasmussen, B. and Fletcher, I.R., 2002, Indirect dating of mafic intrusions by SHRIMP U-Pb analysis of contact metamorphosed shale: An example from the Paleoproterozoic Capricorn Orogen, Western Australia. Earth and Planetary Science Letters, v. 197, pp. 287-299.

Rasmussen, B, Fletcher, I.R. and McNaughton N.J., 2001, Dating low grade metamorphic events by SHRIMP U-Pb analysis of monazite in shales, Geology, v. 29, pp. 963-966.

Rasmussen, B., Fletcher, I.R., Muhling, J.R., Mueller, A.G. and Hall, G.C., 2007, Bushveld-aged fluid flow, peak metamorphism and gold mineralization in the Witwatersand basin, South Africa: Constraints from in-situ SHRIMP U-Pb dating of monazite and xenotime. Geology, v. 35, pp. 931-934.

Rosenblum, S. and Mosier Elwin, L., 1983, Mineralogy and Occurrence of Europium-Rich Dark Monazite. USGS Profressional Paper, 1181, 67 p.

Roy Choudhury, M.K. and Das Gupta, S.P., 1965, Ore-localisation in the Khetri copper belt, Rajasthan, India. Economic Geology, v. 60, pp. 69-88.

Sarkar, S.C., 2000, Crustal evolution and metallogeny in the Eastern Indian Craton. Special Publication, Geological Survey of India, v. 55, pp. 169194.

Sarkar, S.C. and Dasgupta, S., 1980, Geologic setting, genesis and transformation of the sulphide deposits in the northern part of the Khetri Copper Belt, Rajasthan, India - an outline. Mineral Deposita, v. 15, pp. 117-137.

Seydoux-Guillaueme, A.M., Wirth, R., Heinrich, W. and Montel, J.M., 2002, Experimental determination of the Th partitioning between monazite and xenotime using Analytical Electron Microscopy and X-ray Diffraction Rietveld analysis. European Journal of Mineralogy, v. 14, pp. 869-878.

Sharma, R.S., 1988, Patterns of metamorphism in the Precambrian rocks of the Aravalli mountain belt. In: Roy A.B. (ed.) Precambrian of the Aravalli Mountain, Rajasthan, India. Geological Society of India Memoir, no. 7, pp. $33-76$

Singh, S.P., 1984c, Evolution of the Proterozoic Alwar sub-basin, northeastern Rajasthan. Indian Journal Earth Sciences, CEISM, pp. 113-124 
Sinha-Roy, S., Malhotra, G. and Mohanty, M., 1998, Geology of Rajasthan. Geological Society of India, Bangalore, $278 \mathrm{p}$.

Spear, F.S. and Pyle, J.M., 2002, Apatite, monazite and xenotime in metamorphic rocks, in Khn, M.J., Rakovan, J. and Hughes, J.M. (eds.) Phosphates; geochemical, geobiological and materials importance: Reviews in Mineralogy and Geochemistry, v. 48, pp. 293-335.

Townsend, K.J., Miller, C.F., D'Andrea, J.L., Ayers, J.C., Harrison, T.M., and Coath, C.D., 2000, Low temperature replacement of monazite in the Ireteba granite, Southern Nevada: geochronological implications: Chemical Geology, v. 172, pp. 95-112, http://dx.doi.org/10.1016/S00092541(00)00238-2.

Wyhlidal, S., Thony, W.F., Tropper, P. and Mair, V., 2009, Mineralogical and petrological constrains on Permian contact metamorphism at the rims of the Ifinger granodiorite and the Kreuzberg granite (South Tyrol, Italy). Australian Journal of Earth Sciences, v. 102, pp. 181-192.

Wing, B.A., Ferry, J.M., and Harrison, T.M., 2003, Prograde destruction and formation of monazite and allanite during contact and regional metamorphism of pelites: petrology and geochronology. Contribution to Mineralogy and Petrology, v. 145, pp. 228-250, DOI 10.1007/s00410003-0446-1.

Zhu, X.K. and O'Nions, R.K. 1999 Zonation of monazite in metamorphic rocks and its implications for high temperature thermochronology: a case study from the Lewisian terrain. Earth Planetary Science Letters, v. 171, no. 2, pp.209-220. 